\title{
Bilan épidémiologique du paludisme au Maroc
}

\author{
(Données recueillies en 1960, 1961 et 1962)
}

\section{Par Yves GUY}

\section{I. - Les Parasites}

Le Maroc s'apprête à entreprendre l'éradication du paludisme. La phase de prééradication a débuté en 1963. Les années 1960, 1961 et 1962 ont été utilisées à l'étude de l'épidémio'ogie du paludisme.

Jusqu'en 1959 compris, les statistiques du paludisme se basaient non pas sur les cas hématologiquement confirmés mais sur les cas cliniques déclarés qui n'étaient pas obligatoirement confirmés par un laboratoire. Ce n'est que depuis 1960 que les statistiques ne se fondent plus que sur les cas ayant reçu une confirmation parasitologique grâce à l'examen d'une goutte épaisse ou d'un frottis de sang. Depuis cette époque également, les index hématologiques systématiques ont été parachevés.

Jusqu'en 1960 on ne peut donc se baser que sur des approximations : taux de morbidité à 4,9 pour 1000 en 1956 , à 2,19 pour 1000 en $1957 ; 43$ décès par paludisme en 1956,9 décès en 1957 et 9 décès également en 1958.

Des statistiques plus précises font ressortir $(1,3)$ les chiffres suivants qui concernent des cas « constatés » :

$\begin{array}{ll}1934: 168742 & 1943: 292076 \\ 1935: 135176 & 1944: 156858 \\ 1936: 190000 & 1945: 158683 \\ 1937: 138130 & 1946: 260976 \\ 1938: 144398 & 1947: 303740 \\ 1939: 198954 & 1948: 246171 \\ 1940: 176877 & 1949: 233830 \\ 1941: 360264 & 1950: 151807 \\ 1942: 286084 & 1951: 145052\end{array}$

$1952: 88716$

Par la suite, les rapports annuels des formations indiquent les chiffres suivants :

$1953: 82600$

1954: 49085

1955: 34767
1956: 97599

1957: 55425

1958: 43393

$1959: 42319$ 


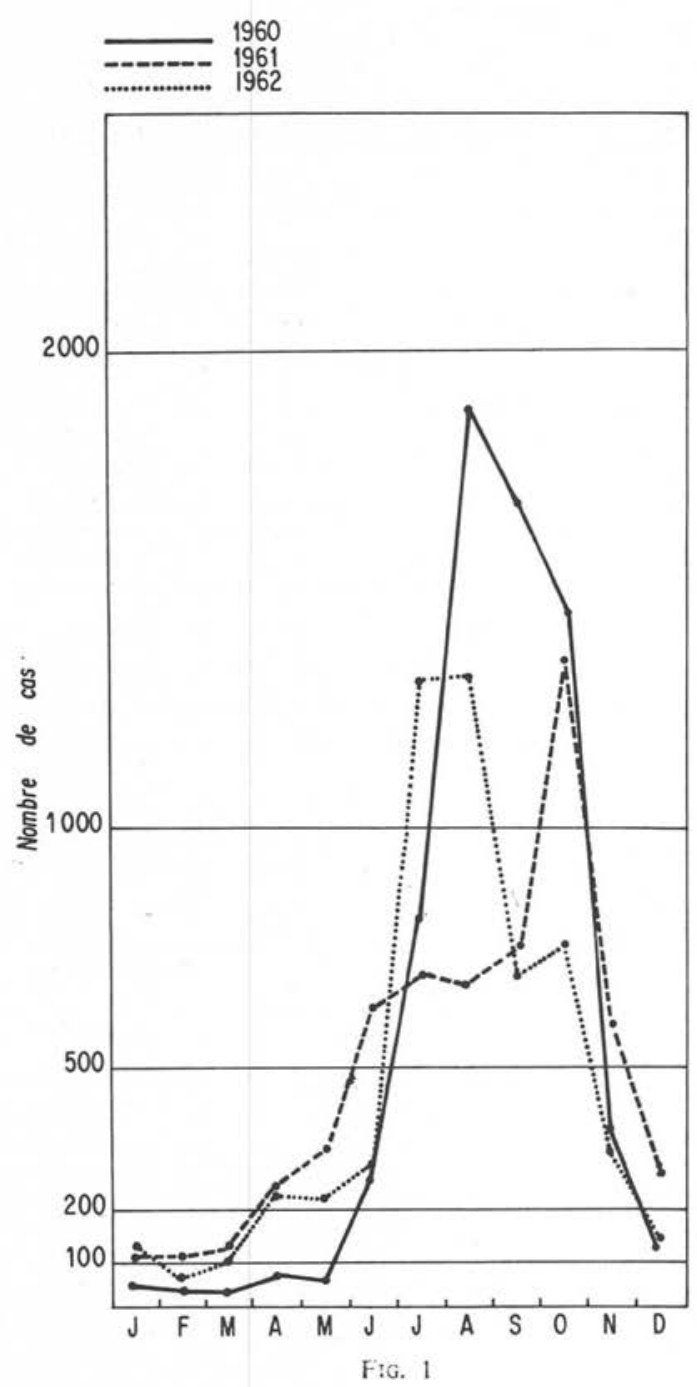

Les résultats obtenus en 1960, 1961 et 1962 montrent que le paludisme est hypo-endémique, mais qu'il existe partout au Maroc.

Les courbes des trois années ne sont pas strictement comparables (1960 a été une année très sèche et 1962 une année relativement sèche). On peut cependant se rendre compte (fig. 1) que c'est au mois de juin que la courbe des cas devient brusquement ascendante et l'on peut considérer que les cas apparus de janvier à mai compris sont des rechutes (cf. aussi fig. 4 concernant $P$. falciparum). Ce fait est confirmé par l'étude du rapport larves/adultes d'Anophèles (2). De même, la figure 6 montre que les cas à $P$. malariae, d'apparition plus tardive, ne débutent d'une façon - conséquente, sous forme de primo-infections, qu'en juillet.

Le paludisme est à son maximum pendant les mois de juillet à octobre et la courbe descend en novembre.

Pour l'ensemble du Maroc, la période de transmission du paludisme s'étend donc de mai à octobre. 


\section{Indice systématique.}

En premier lieu, on peut effectivement conclure de l'indice hématologique pratiqué sur les enfants d'âge scolaire qu'aucune région du Maroc n'est indemne de paludisme. Des indices élevés se rencontrent précisément dans les régions traditionnellement considérées comme non impaludées (Ouarzazate et Tafilalet, au Sud du Haut-Atlas), ce qui revient à dire que la totalité de la population rurale du pays devra être protégée par les insecticides à effet rémanent, soit plus de 9 millions de personnes.

Le résultat de cet indice est représenté sur le tableau I.

Cas confirmés par laboratoire (contrôles de diagnostic).

28493 lames ont été examinées en 1960, 43932 en 1961 et 87697 en 1962.

Lorsqu'on considère les cas confirmés par laboratoire, on constate (fig. 1) :

TABleau I

\begin{tabular}{|c|c|c|c|c|c|c|}
\hline Provinces & $\begin{array}{l}\text { Population } \\
\text { rurale }\end{array}$ & $\begin{array}{c}\text { Nombre } \\
\text { de } \\
\text { lames } \\
\text { prélevées }\end{array}$ & $\begin{array}{l}\% \text { de } \\
\text { lames } \\
\text { prélevées } \\
\text { par } \\
\text { rapport } \\
\text { à la } \\
\text { popula- } \\
\text { tion } \\
\text { rurale }\end{array}$ & $\begin{array}{c}\text { Nombre } \\
\text { de } \\
\text { lames } \\
\text { positives }\end{array}$ & $\begin{array}{c}\text { Indice } \\
\% 0\end{array}$ & $\begin{array}{l}\text { Paludéens } \\
\text { probables }\end{array}$ \\
\hline Agadir ........ & 829176 & 32100 & $3,5 \%$ & 11 & 0,03 & 284 \\
\hline Oujda, Nador .. & 732687 & 31895 & $4,3 \%$ & 24 & 0,07 & 550 \\
\hline Beni Mellal .... & 476392 & 19336 & $4,0 \%$ & 27 & 0,13 & 664 \\
\hline Tétuan ........ & 459851 & 23535 & $5,1 \%$ & 41 & 0,17 & 800 \\
\hline Taza $\ldots . . \ldots$ & 412741 & 13461 & $3,2 \%$ & 35 & 0,25 & 1050 \\
\hline Fès, Al Hucei na & 773900 & 39170 & $5,0 \%$ & 158 & 0,38 & 3002 \\
\hline Rabat $\ldots \ldots$. & 934616 & 36766 & $3,9 \%$ & 142 & 0,38 & 3607 \\
\hline Marrakech .... & 1641188 & 61938 & $3,8 \%$ & 293 & 0,47 & 7845 \\
\hline Casablanca .... & 1222845 & 53672 & $4,3 \%$ & 307 & 0,56 & 7010 \\
\hline Tafilalet $\ldots .$. & 387672 & 18585 & $4,9 \%$ & 208 & 1,1 & 4326 \\
\hline Meknès ....... & 391139 & 21155 & $5,4 \%$ & 277 & 1,3 & 5097 \\
\hline Ouarzazate .... & 434122 & 15933 & $3,7 \%$ & 543 & 3,4 & 14770 \\
\hline Total ....... & 8215433 & 367546 & $4,4 \%$ & 2066 & 0,5 & 46103 \\
\hline
\end{tabular}




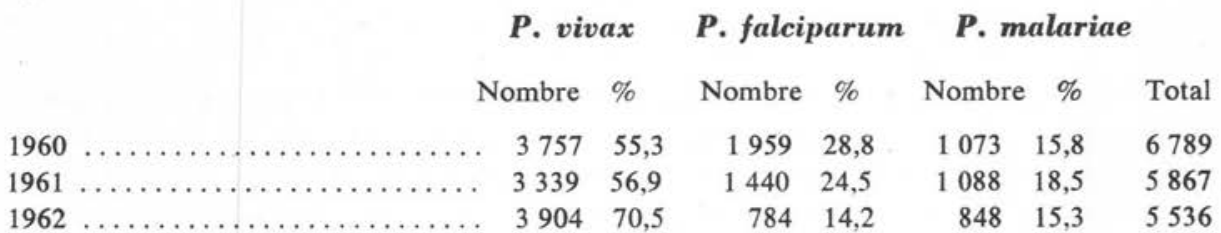

Le ca!endrier s'établit ainsi :

$1^{\circ}$ Plasmodium vivax (fig. 2 et 3 ):

\begin{tabular}{|c|c|c|c|}
\hline & 1960 & 1961 & 1962 \\
\hline Janvier & $13(38,2 \%)$ & $79(74,5 \%)$ & $70(58,8 \%)$ \\
\hline Février & $19(61,3 \%)$ & $82(82,0 \%)$ & $45(84,9 \%)$ \\
\hline Mars . & $23(74,2 \%)$ & $103(83,7 \%)$ & $79(87,7 \%)$ \\
\hline Avril & $48(90,5 \%)$ & $208(79,6 \%)$ & $195(83,6 \%)$ \\
\hline Mai $\ldots$. & $29(60,4 \%)$ & $235(73,4 \%)$ & $187(75,1 \%)$ \\
\hline Juin .. & $150(58,3 \%)$ & $389(62,9 \%)$ & $195(63,7 \%)$ \\
\hline Juillet & $370(47,5 \%)$ & $419(60,6 \%)$ & $862(66,6 \%)$ \\
\hline Août $\ldots$ & $1004(54,5 \%)$ & $329(49,2 \%)$ & $907(69,7 \%)$ \\
\hline Septembre & $927(52,5 \%)$ & $341(44,9 \%)$ & $441(66,1 \%)$ \\
\hline Octobre $\ldots$ & $914(62,0 \%)$ & $731(53,2 \%)$ & $566(74,6 \%)$ \\
\hline Novembre . & $197(55,0 \%)$ & $280(46,3 \%)$ & $262(80,1 \%)$ \\
\hline Décembre ..... & $63(50,4 \%)$ & $143(58,1 \%)$ & $95(67,8 \%)$ \\
\hline Total & $3757(55,3 \%)$ & $339(56,9 \%)$ & $904(70,5 \%$ \\
\hline
\end{tabular}

$2^{\circ}$ Plasmodium falciparum (fig. 4 et 5):

1960

1961

1962

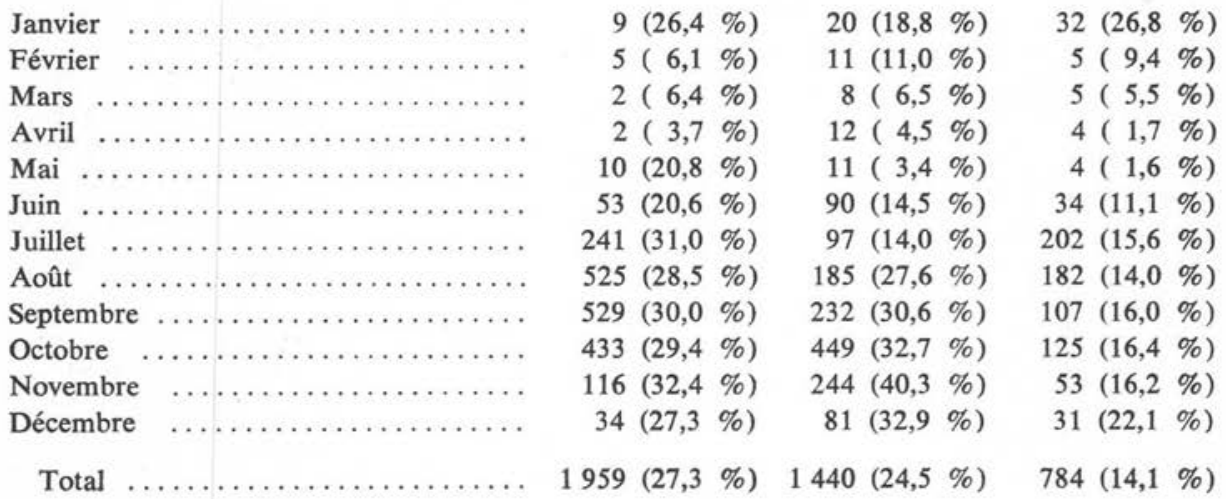


$3^{\circ}$ Plasmodium malariae (fig. 6 et 7 ):

1960

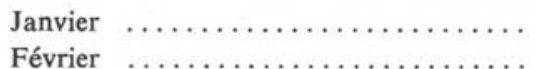

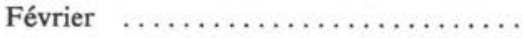

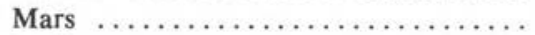

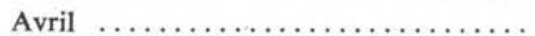

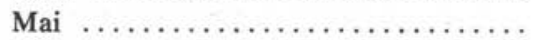

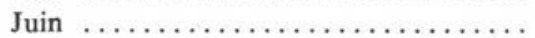

Juillet $\ldots \ldots \ldots \ldots \ldots \ldots \ldots \ldots \ldots \ldots \ldots \ldots$

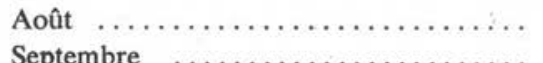

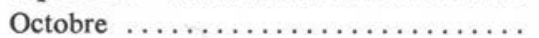

Novembre $\ldots \ldots \ldots \ldots \ldots \ldots \ldots \ldots$

Décembre

Total
1961

1962

$\begin{array}{rrrr}12(35,2 \%) & 7(6,6 \%) & 17(14,2 \%) \\ 7(22,5 \%) & 7(7,0 \%) & 3(5,6 \%) \\ 6(19,3 \%) & 12(9,7 \%) & 6(6,6 \%) \\ 3(5,6 \%) & 41(15,7 \%) & 34(14,5 \%) \\ 9(18,7 \%) & 74(23,1 \%) & 58(23,2 \%) \\ 54(21,0 \%) & 139(22,4 \%) & 77(25,1 \%) \\ 167(21,4 \%) & 175(25,3 \%) & 230(17,7 \%) \\ 312(16,9 \%) & 154(23,0 \%) & 211(16,2 \%) \\ 310(17,5 \%) & 185(24,4 \%) & 119(17,8 \%) \\ 121(8,2 \%) & 192(13,9 \%) & 67(8,8 \%) \\ 44(12,3 \%) & 80(13,2 \%) & 12(3,6 \%) \\ 28(22,4 \%) & 22(8,9 \%) & 14(12,0 \%)\end{array}$

$1073(15,8 \%) \quad 1088(18,5 \%) \quad 848(15,3 \%)$

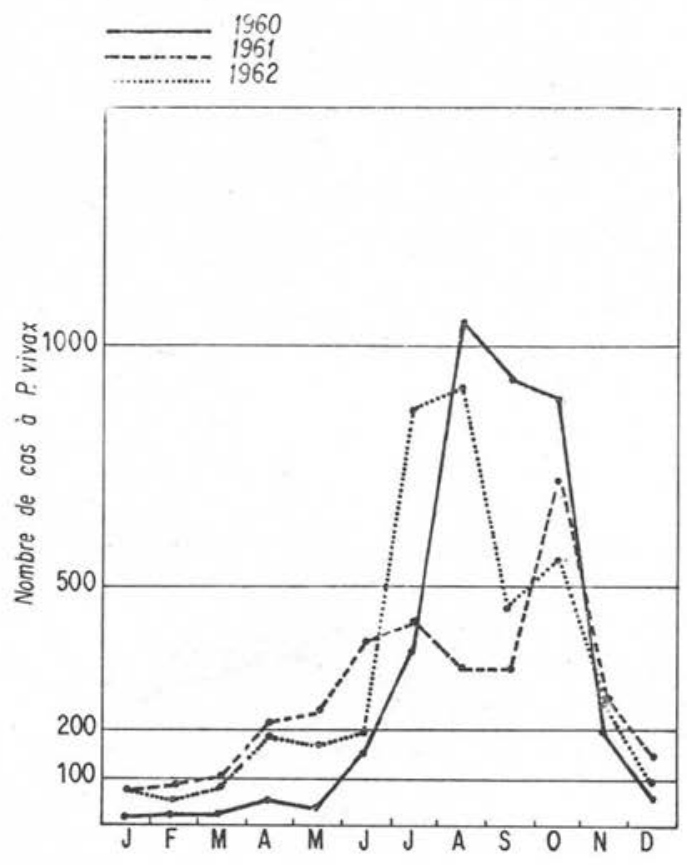

Fig. 2

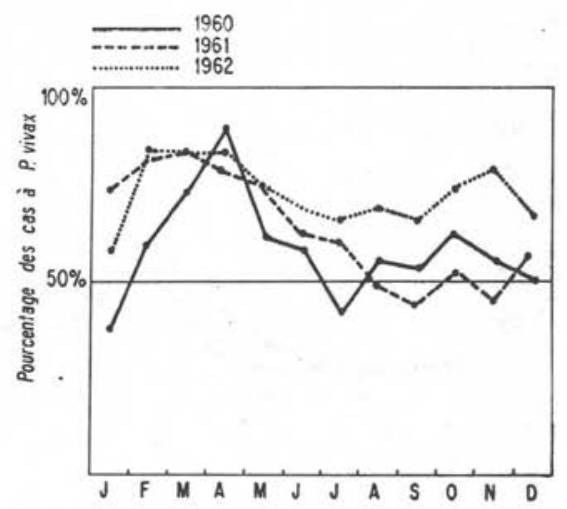

FIG. 3 


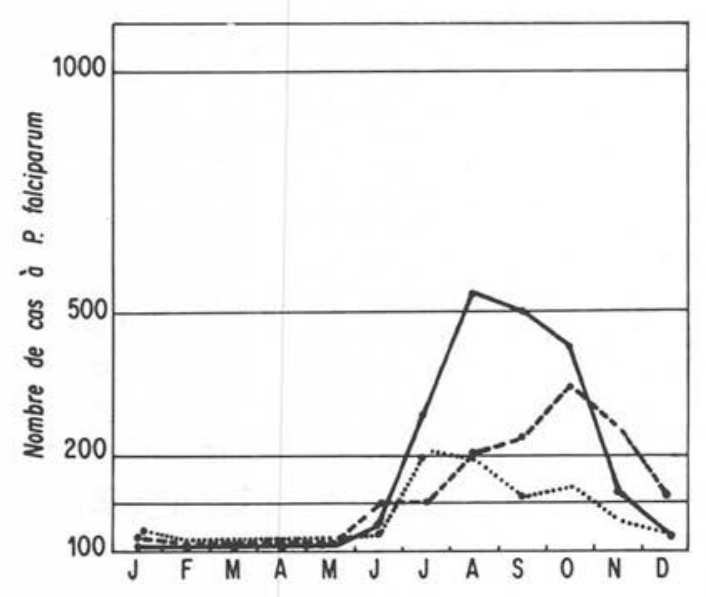

FIG. 4

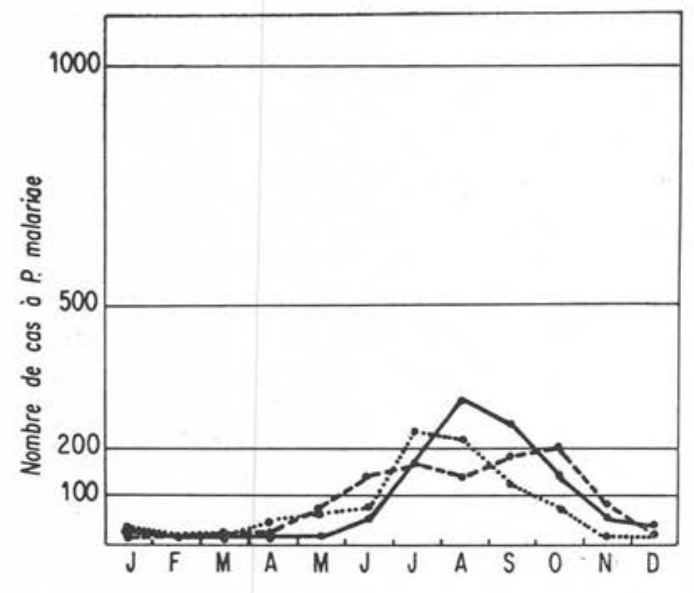

F1G. 6

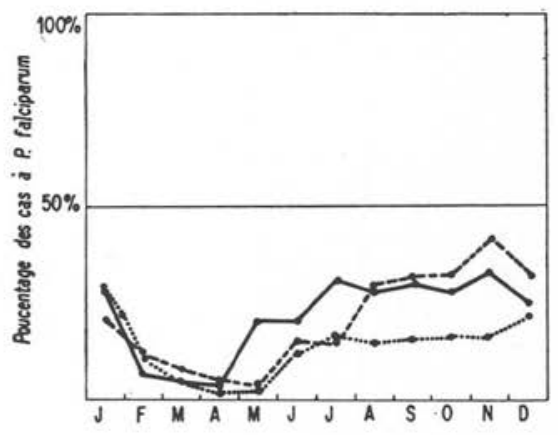

FIG. 5

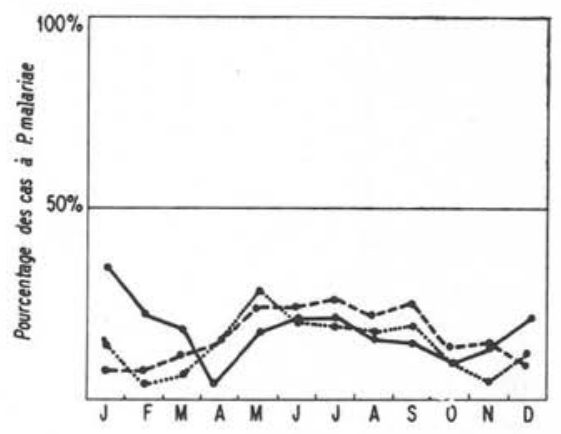

Fig. 7 
On peut tenter d'établir un bilan de l'évolution générale de la situation avant de passer à une étude plus détaillée de l'épidémiologie. Il est assez difficile pourtant de faire des comparaisons valables avec les années antérieures à 1960 car il s'agissait avant cette date non pas de cas confirmés mais de " cas constatés » dans des formations fixes.

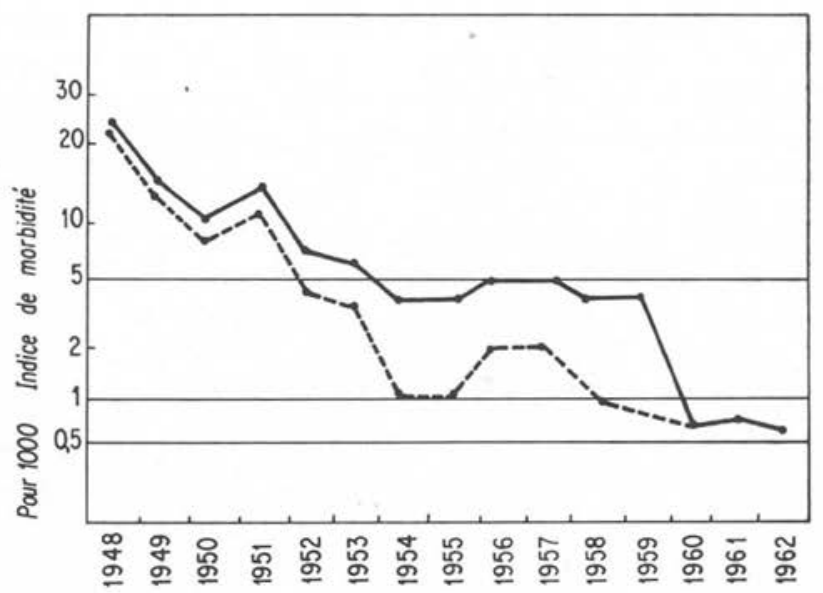

Fig. 8

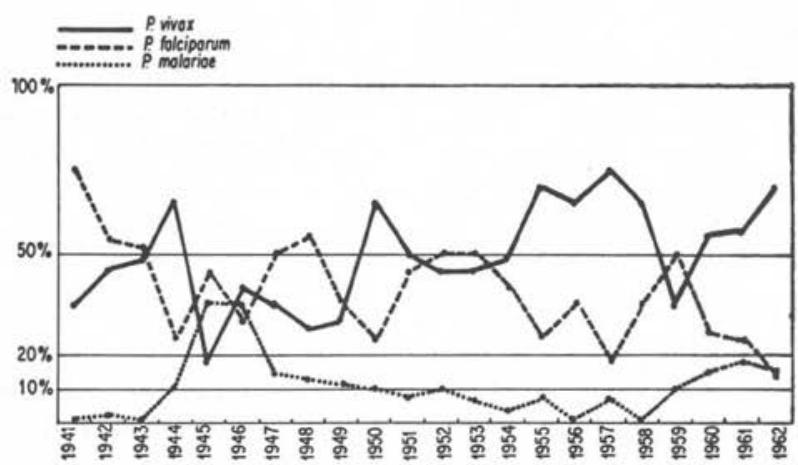

Fig. 9

Cependant, si l'on garde cette notion présente à l'esprit, on peut établir une courbe de l'indice de morbidité qui montre malgré tout la décrue des cas (fig. 8). La courbe pointillée de la figure 8 a été tracée en considérant que l'année 1955 est à peu près semblable à l'année 1960 : ce sont deux années normales aussi bien du point de vue épidémiologique qu'entomologique et climatique. L'excès de cas constatés en 1955 par rapport aux cas confirmés de 1960 est donc de 2,9 et l'on soustrait ce coefficient aux indices de morbidité successifs, ce qui permet le tracé de la courbe corrigée (en pointillé). 
L'indice de morbidité pour 1.000 personnes est à 0,6 en $1960,0,7$ en 1961 et à nouveau 0,6 en 1962. Il s'agit donc d'un paludisme hypoendémique et instable (cf. fig. 1 à 7). On voit mieux sur la courbe corrigée que la décrue des cas est régulière depuis 1948 avec cependant une poussée plus forte en 1951, puis en 1956 et 1957 (années à pluviométrie importante).

Enfin, la figure 9 montre que pour 1.000 lames positives, le pourcentage des cas dûs à $P$. falciparum a tendance à diminuer alors que celui des cas dus à $P$. vivax a tendance à augmenter : c'est un phénomène normal dans un pays contrôlé par une lutte anti-paludique. Le pourcentage des cas dûs à $P$. malariae a également tendance à diminuer.

Lorsqu'on considère les cas de paludisme survenus suivant les groupes d'âges, on retrouve les pourcentages suivants pour l'année 1962 :

\begin{tabular}{|c|c|c|c|c|c|}
\hline & $0-1$ an & $1-4$ ans & $5-9$ ans & $10-14$ ans & +15 ans \\
\hline Tétouan & $\%$ & $5,76 \%$ & $13,47 \%$ & $7,69 \%$ & $73,07 \%$ \\
\hline Rabat & $3,38 \%$ & $16,10 \%$ & $18,48 \%$ & $14,45 \%$ & $47,57 \%$ \\
\hline Meknès & $1,11 \%$ & $12,04 \%$ & $19,89 \%$ & $14,17 \%$ & $52,77 \%$ \\
\hline Fès & $0,93 \%$ & $9,97 \%$ & $16,90 \%$ & $15,49 \%$ & $56,69 \%$ \\
\hline Marrakech .... & $1,85 \%$ & $15,63 \%$ & $20,78 \%$ & $11,72 \%$ & $50,00 \%$ \\
\hline Tafilalet $\ldots \ldots \ldots \ldots \ldots \ldots$ & $1,09 \%$ & $4,39 \%$ & $15,93 \%$ & $19,78 \%$ & $58,79 \%$ \\
\hline
\end{tabular}

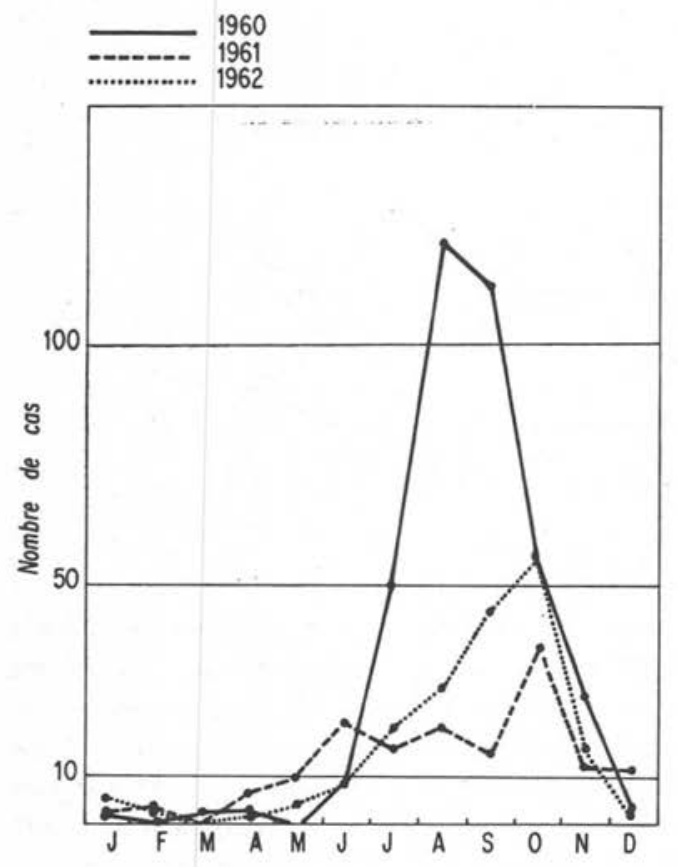

On voit qu'en majorité les cas sont contractés après 15 ans. C'est ensuite de 5 à 9 ans que se rencontrent le plus de cas (sauf au Tafilalet) alors que de 10 à 14 ans les enfants sont proportionnellement moins atteints. Enfin, les enfants de 0 à 4 ans (et surtout de 0 à 1 an) sont les moins atteints. Ces données, si différentes de celles que l'on peut constater en Afrique de climat équatorial par exemple confirment bien que la transmission est discontinue au cours de l'année et que l'immunité acquise ne peut pas s'établir en raison de la rareté relativl des possibilités d'infestation.

FIG. 10 


\section{Division du Maroc en zones épidémiologiques.}

\section{Zone côtière méditérranéenne.}

Celle-ci se compose (cf. carte) des :

- Province de Tanger,

- Province de Tétouan : cercles de Jébala, Tétouan, Baria et Chaouen,

- Province d'Al Huceima,

- Province de Nador,

- Province d'Oujda : cercles de Berkane et Oujda.

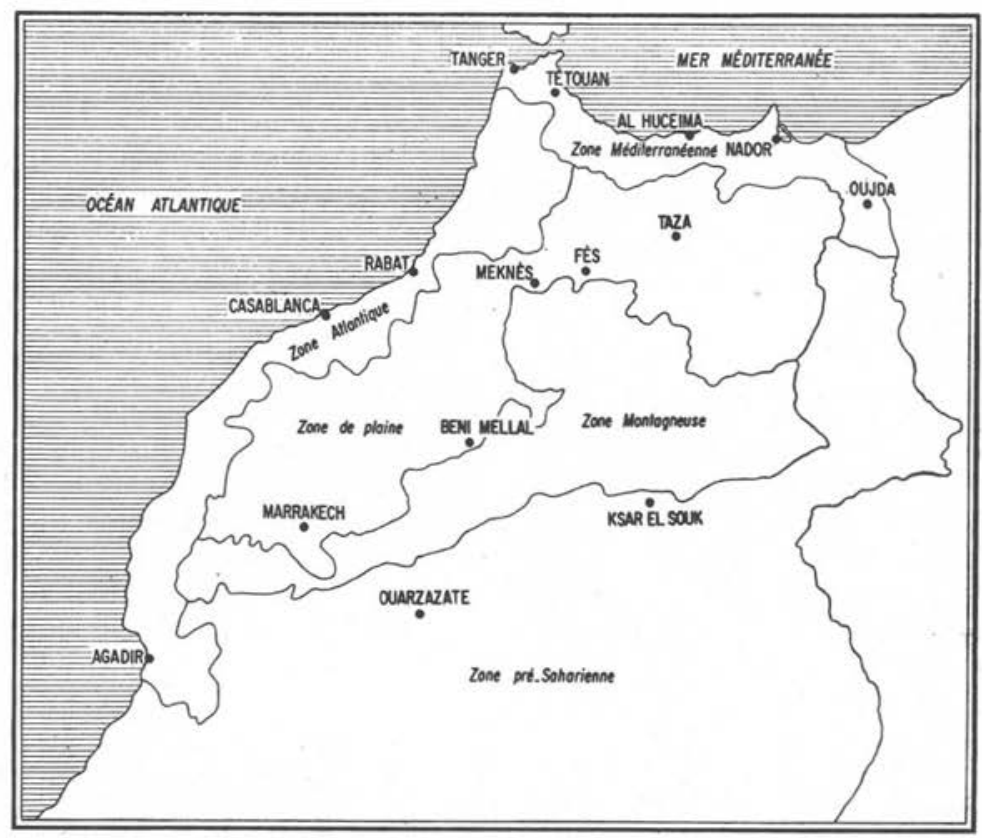

L'ascension de la courbe des cas se produit entre mai et juillet suivant les années (fig. 10) et le maximum se situe en août, septembre et octobre. On voit ici une différence que l'on rencontrera dans la presque totalité du Maroc: le nombre de cas a été plus élevé en 1960 que pendant les deux autres années et c'est l'année 1961 (à pluviométrie normale en chiffre absolue, mais groupée au seul printemps) qui a connu le moins de cas. On compte ainsi 389 cas dans cette zone méditerranéenne en 1960, 166 en 1961 et 186 en 1962 pour 1.458 lames prélevées en $1960,4.336$ en 1961 et 15.161 en 1962 et pour une population rurale de 1.131 .522 personnes. C'est la zone la moins impaludée du Maroc.

La province de Tanger (22.600 habitants ruraux) est très peu impaludée : 19 cas 
en 1960 (260 lames prélevées), 6 cas en 1961 (1.840 lames prélevées) et 2 cas en 1962 (530 lames prélevées).

Les provinces d'Oujda et Nador sont également peu impaludées : 32 cas en 1960 (200 lames prélevées), 11 cas en 1961 (500 lames prélevées) et 58 cas en 1962 (3.700 lames prélevées) pour une population rurale de 732.687 habitants.

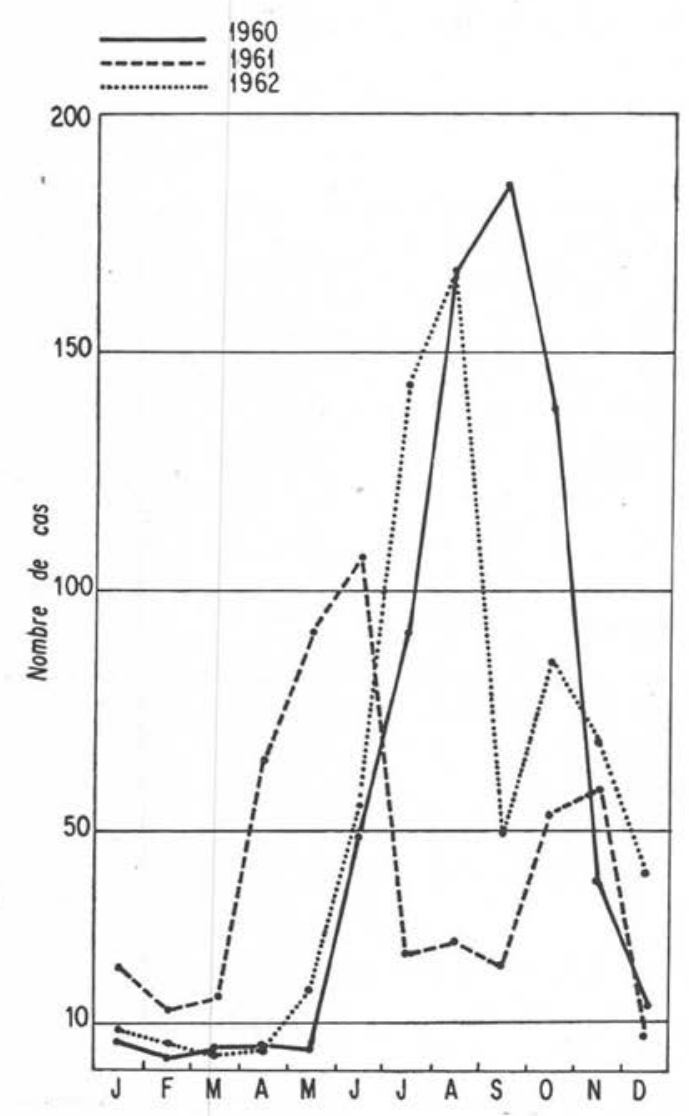

Fig. 11

\section{$2^{\circ}$ Zone côtière atlantique.}

Elle comprend :

- Province de Tétouan: cercles d'Asilah et Larache,

- Province de Rabat: cercles de Souk-el-Arba, Kénitra et Rabat,

- Province de Casablanca : cercles de Chaouïa-Nord (Casablanca), ChaouïaCentre, Azemmour et El Jedida,

- Province de Marrakech : cercles de Safi et Essaouira,

- Province d'Agadir: cercles et Inezgane. Bien que les cercles de Tiznit et Goulimine s'ouvrent sur l'Océan, ils sont cependant comptés dans la zone pré-saharienne en raison du climat plus semblable et du petit nombre d'habitants vivant sur la côte inhospitalière. On ne possède encore que des renseignements très fragmentaires sur la Province de Tarfaya, à l'extrême sud du pays.

Cette zone a été plus influencée que la précédente par les variations climatiques des trois dernières années. C'est ainsi que si la brusque montée des cas s'est produite en juin en 1960 avec le maximum de cas en août, septembre et octobre (fig. 11), en 1961 les pluies printanières importantes sont suivies de la montée des cas en avril, mai et juin (les premières primo-infections apparaissent simultanément avec les rechutes) et un deuxième sommet, automnal, se voit en octobre et novembre ; cette notion d'épidémie tardive (actuellement réduite à de simples poussées) est classique dans cette zone du Maroc. Elle s'objective mieux si l'on dresse le graphique de la distribution mensuelle des cas par rapport au nombre total annuel de cas (fig. 12). La transmission sans interruption au cours de l'été 1960 masque la première diminution naturelle des cas, bien visible en 1961 et 1962, et le 
départ de la courbe des cas en 1962 est très semblable à celui de 1960. Cette deuxième pointe se répartit sur l'ensemble de la zone, mais ce sont les cercles de Casablanca inclus dans cette zone qui en sont les principaux responsables. Enfin en 1962, année intermédiaire en ce qui concerne la pluviométrie, les cas commencent à croître en juin (comme en 1960), sont à leur maximum en juillet et août et réforment une pointe en octobre-novembre (comme en 1961).

On compte ainsi 714 cas pour cette zone en 1960 (2.931 lames prélevées), 508 en 1961 (7.921 lames prélevées) et 660 en 1962 (15.483 lames prélevées) pour une population rurale de 1968581 habitants.

La saison de transmission va habituellement de mai à octobre. La zone côtière atlantique est plus impaludée que la zone côtière méditerranéenne (fig. 13). Le fonds parasitaire (3-4 premiers mois de l'année) est cependant approximativement le même mais les conditions diffèrent dans les deux zones: abondance de marécages dans la zone atlantique, rareté dans la zone méditerranéenne, présence d'Anopheles labranchiae, le grand vecteur marocain dans la première zone, présence d'un mauvais vecteur dominant, $A$. hispaniola, dans la seconde. Cela explique que l'on

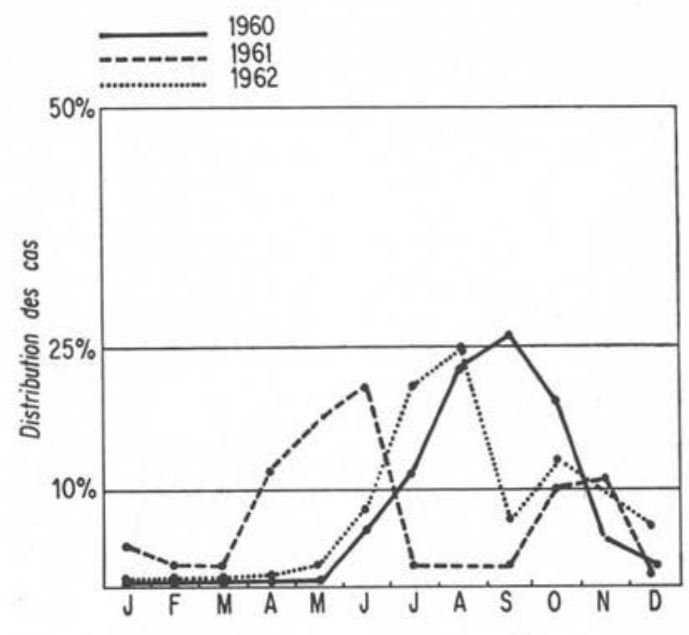

FiG. 12 ait jugé nécessaire d'entreprendre une lutte imagicide depuis une dizaine d'années dans la zone atlantique, alors qu'il n'en a jamais été question sur les rivages de la Méditerranée.

\section{Zone de plaine continentale.}

Elle comprend :

- Province de Rabat : cercles de Ouezzane, Khemisset et Romman,

- Province de Casablanca : cercles de Chaouïa-Sud, Sidi-Bennour, El Khemis des Zemamra, Ben Ahmed, Khouribga et Oued-zem,

- Province de Meknès : cercles de Meknès-banlieue et Khénifra,

- Province de Fès : cercles de Karia-ba-Mohamed, Taounate et Fès-banlieue,

- Province de Taza,

- Province d'Oujda: cercle de Taourirt,

- Province de Beni-Mellal : cercles de Fquih-ben-Salah et Beni-Mellal, des Shrarna.

- Province de Marrakech : cercles de Chemaïa, Marrakech-banlieue, Rehamma, El Kelaa 
Ci-contre: FIG. 13
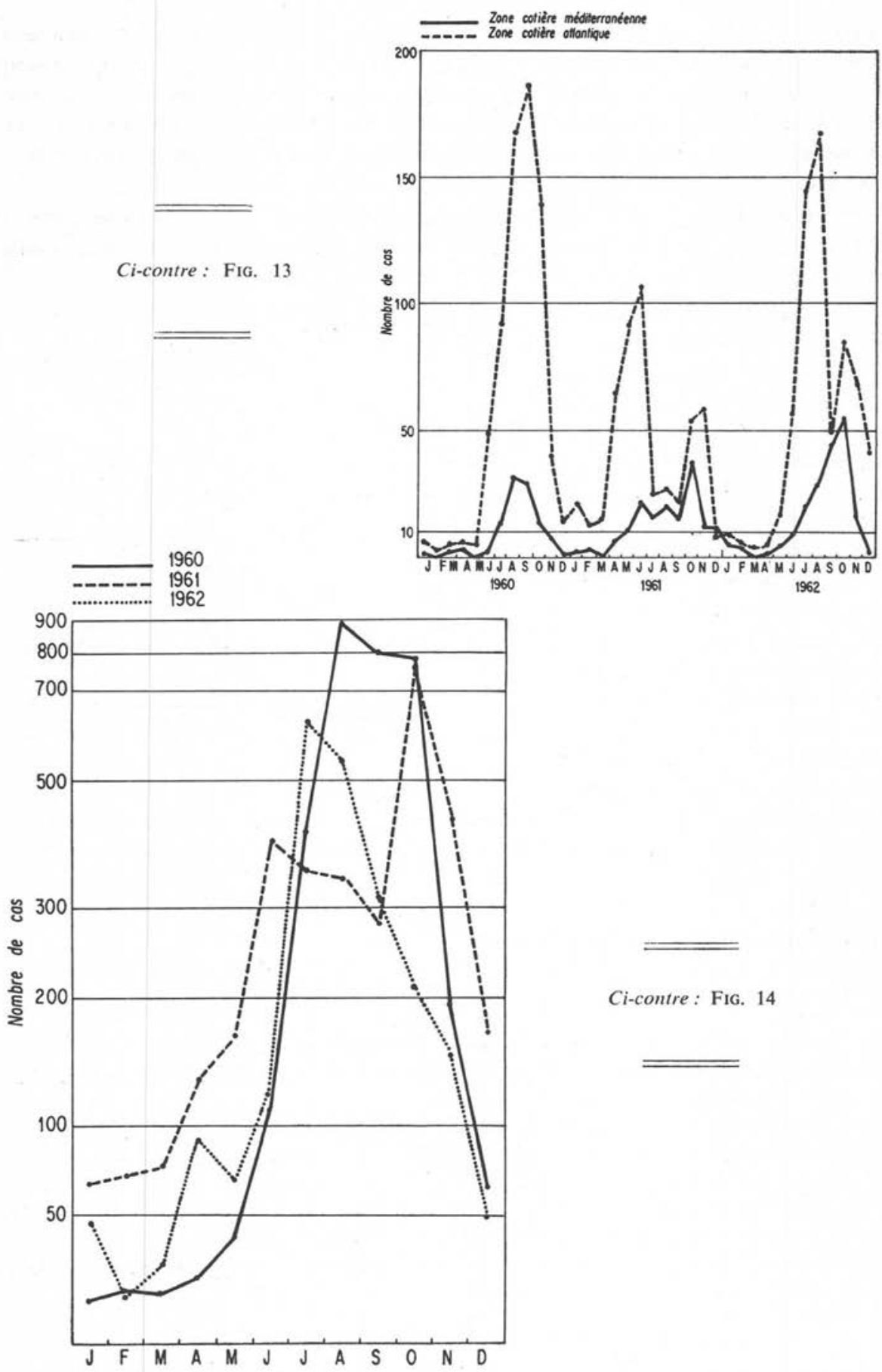

Ci-contre: FIG. 14 


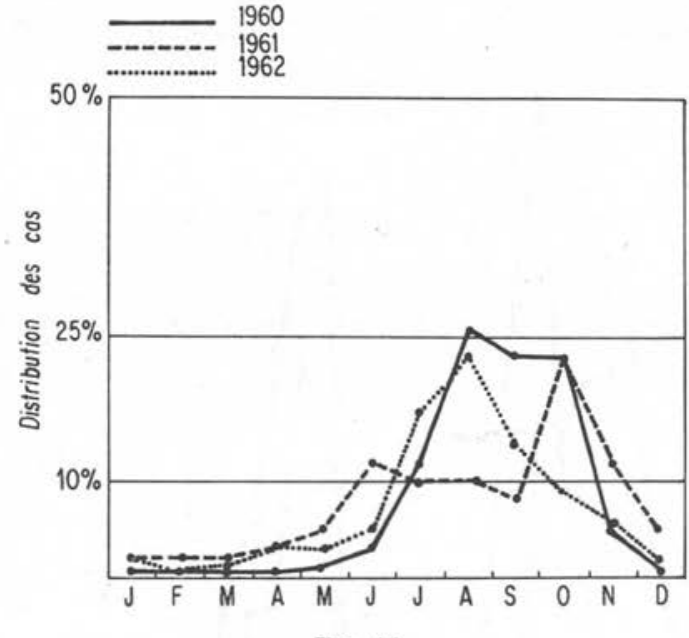

Fig. 15

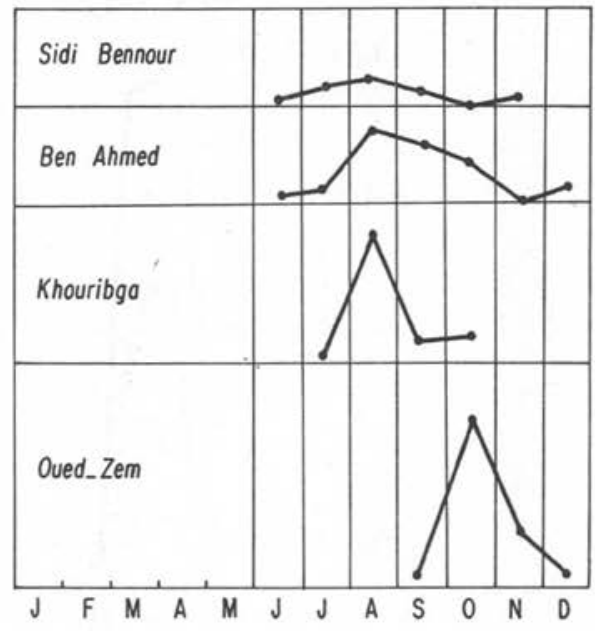

Fig. 16

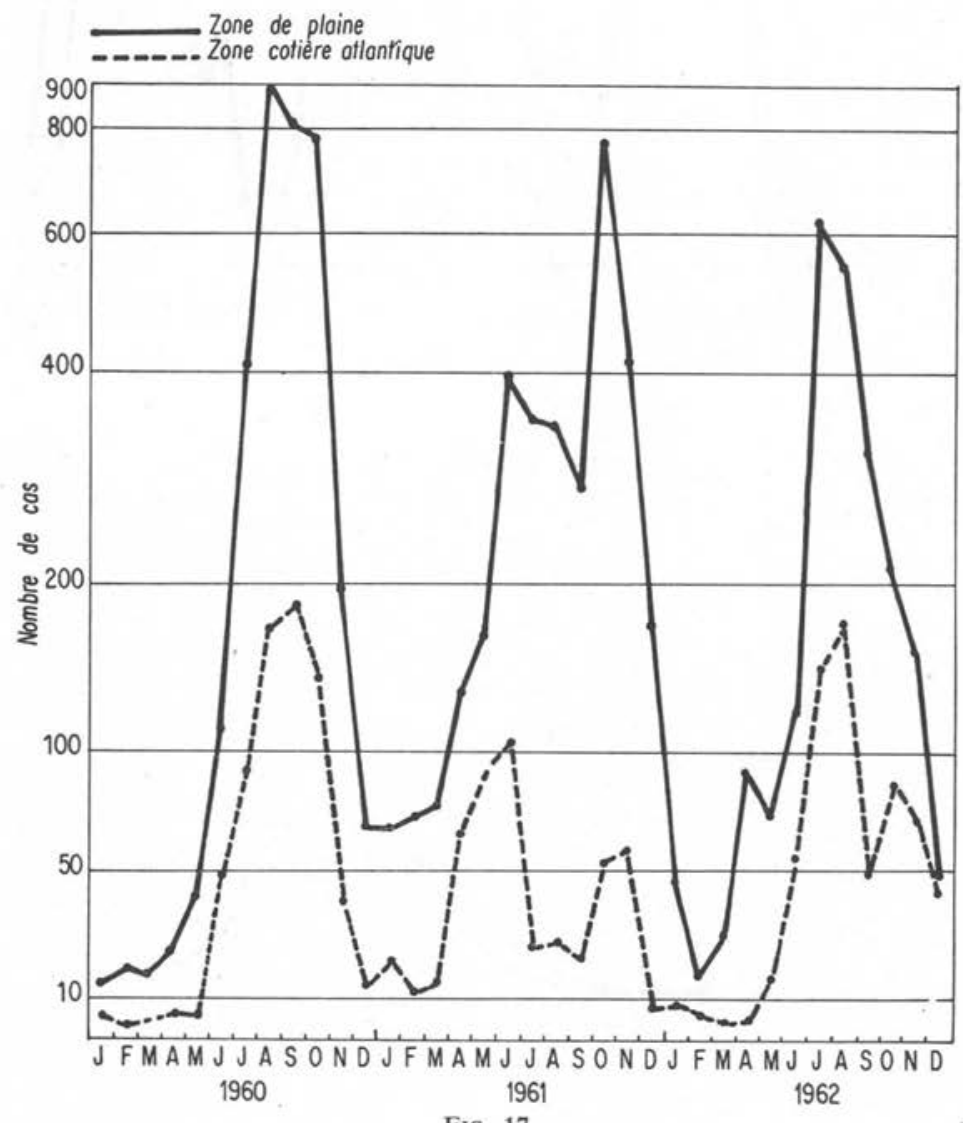

Fig. 17 


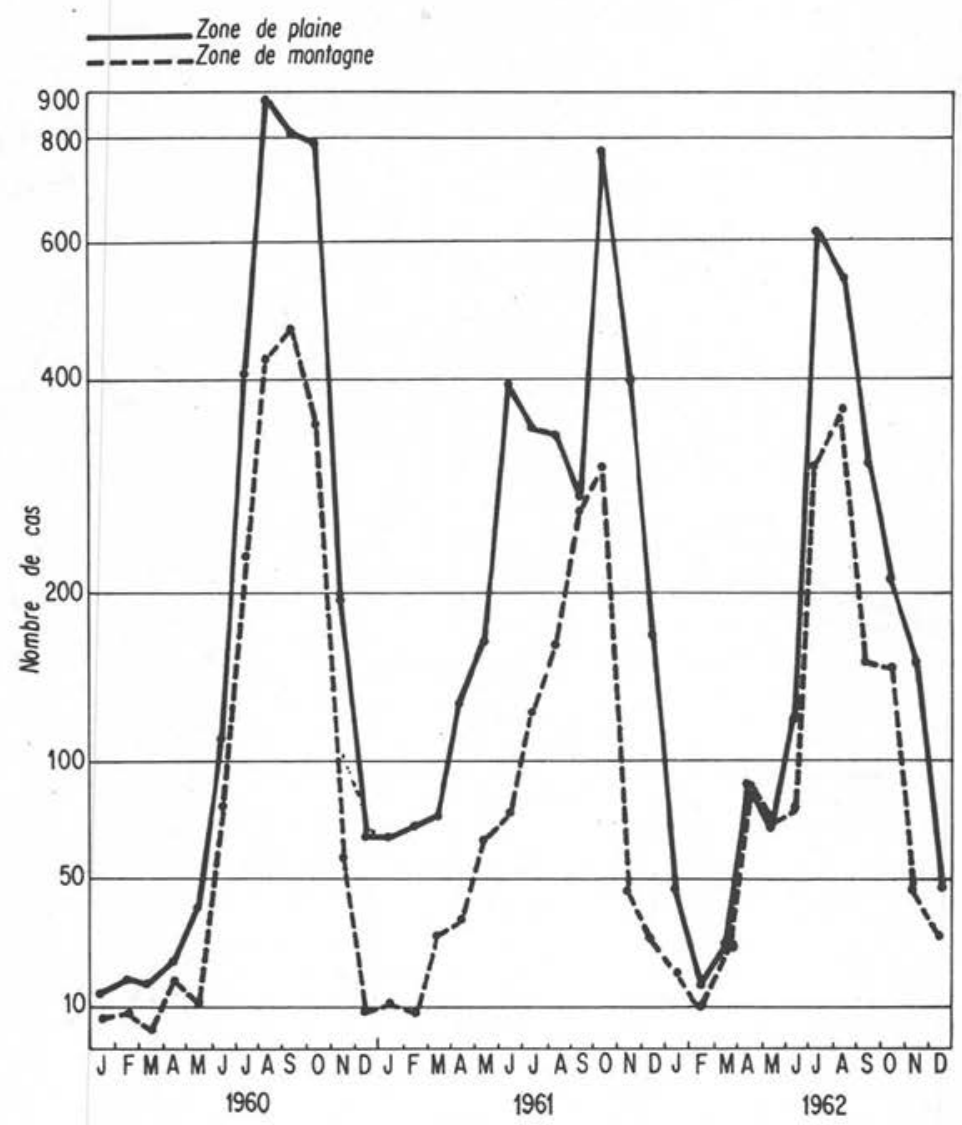

Fig. 18

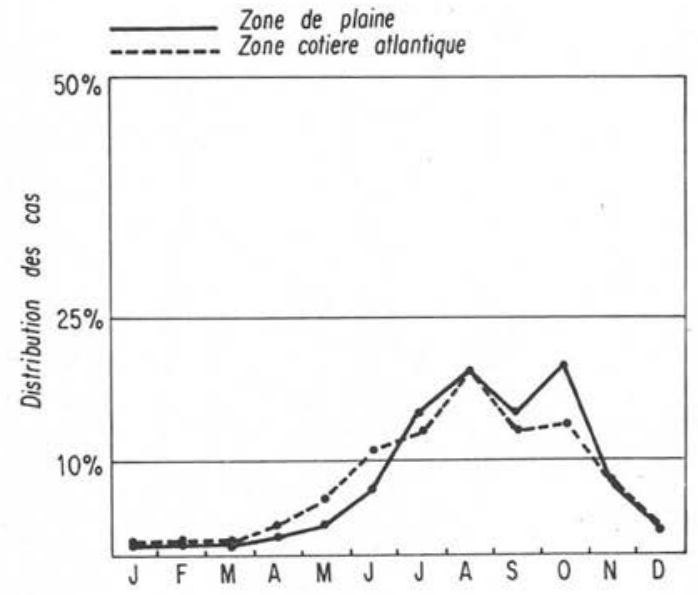

FIG. 19 
La plaine marocaine, très étendue, représente la zone la plus impaludée du Maroc et en même temps la plus peuplée (3 139858 habitants). On y comptait 3379 paludéens en 1960 (11 581 lames prélevées), 3227 en 1961 (15739 lames prélevées) et 2253 en 1962 (19668 lames prélevées).

La courbe est beaucoup plus régulière, moins influencée par les variations climatiques et pluviométriques annuelles (fig. 14), que dans la dernière zone. On note que le fonds parasitaire est relativement important: lorsque la courbe descend, il persiste 66 cas en décembre 1960, 170 en décembre 1961 et 48 en décembre 1962. Cependant la répartition des cas (fig. 15) et assez semblable à elle-même (bien que, pour la même raison que dans les premières zones, la courbe de 1961 en diffère sensiblement). La courbe des cas débute en mai plutôt qu'en juin et persiste jusqu'en novembre. La période de transmission est donc globalement d'une durée plus longue que sur la côte et le paludisme est plus «pur » dans cette zone que dans la précédente, seul le cercle d'El Kelaa des Shrarna (province de Marrakech) ayant connu une lutte imagicide pendant ces quatre dernières années.

Plus l'on s'enfonce de la côte vers l'intérieur, plus le paludisme est tardif et plus il est important. La figure 16 en donne un exemple tiré de la province de Casablanca en 1960. La même année, on retrouve dans cette même province une courbe avec un sommet en août et un autre en octobre (en 1961 les deux sommets sont respectivement en septembre et novembre ; ils sont à nouveau en août et en octobre en 1962) : ce sont les cercles les plus près de la bande côtière dont le maximum de cas est responsable du premier sommet tandis que les cercles les plus continentaux sont responsables du $2^{\circ}$ sommet.

Le même phénomène se retrouve lorsqu'on considère non plus une province isolée mais l'ensemble de la zone de plaine: plus l'on s'écarte de la côte, plus les cas sont nombreux. Il y a davantage de cas dans la plaine de la province de Meknès, que dans la plaine des provinces de Casablanca ou Rabat, davantage dans la province de Fès, que dans celle de Meknès. Puis, toujours en allant vers l'Est, le phénomène s'inverse : il y a moins de cas dans la province de Taza, que dans la province de Fès et moins dans la province d'Oujda que dans celle de Taza.

La figure 17 rend compte de ces phénomènes et permet de constater que la plaine marocaine est plus impaludée que la zone côtière atlantique (qui connaît en grande partie une lutte imagicide ; il est traditionnel de dire qu'avant celle-ci, c'était le contraire qui se produisait); que la plaine connaît une période du paludisme et une période de transmission plus étendues sur l'année et que le fonds parasitaire persistant en fin d'année y est plus important. Par contre, on peut se rendre compte (fig. 18), que la répartition mensuelle des cas est à peu près semblable dans les deux zones.

\section{Zone montagneuse (Haut-Atlas et Moyen-Atlas).}

Elle se compose de :

- Province de Fès : cercles de Sefrou et Boulemane,

- Province de Meknès : cercle d'El Hajeb et Azrou, 


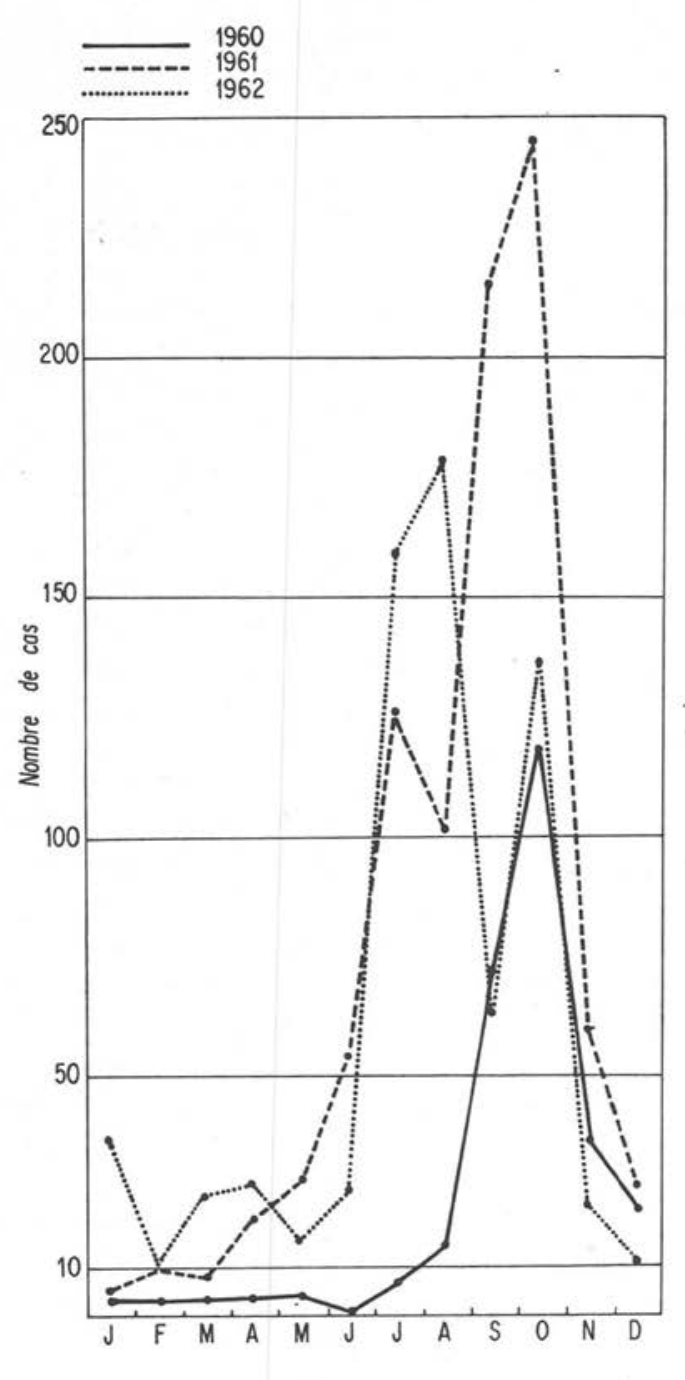

FIG. 20

- Province de Beni-Mellal: cercles d'El Ksiba, Ouaouizerth et Azilal,

- Province de Ksar el Souq (Tafilalet) : cercles de Midelt et Rich,

- Province de Marrakech : cercles d'Imi n'Tanoute, Amizmiz et Aït Ourir.
La montagne marocaine est impaludée : 1695 cas en 1960 (5958 lames prélevées), 1164 cas en 1961 (8071 lames prélevées) et 1056 cas en 1962 (15 270 lames prélevées) pour une population rurale de 1141264 habitants.

Du point de vue épidémologique, cette zone ne présente que peu de différences avec la plaine (fig. 19), mais le nombre résiduel de cas en fin d'année y est moins important.

\section{Zone pré-saharienne.}

Elle se compose de :

- Province d'Agadir : cercles de Taroudant, Tiznit et Goulimine,

- Province de Ouarzazate,

- Province de Ksar el Souq: cercles de Goulmina, Ksar el Souq et Erfoud.

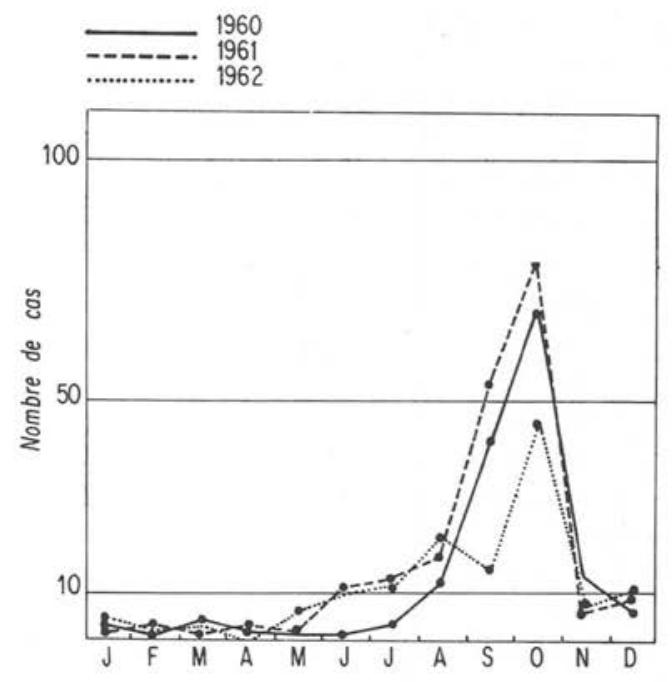

FIG. 21 


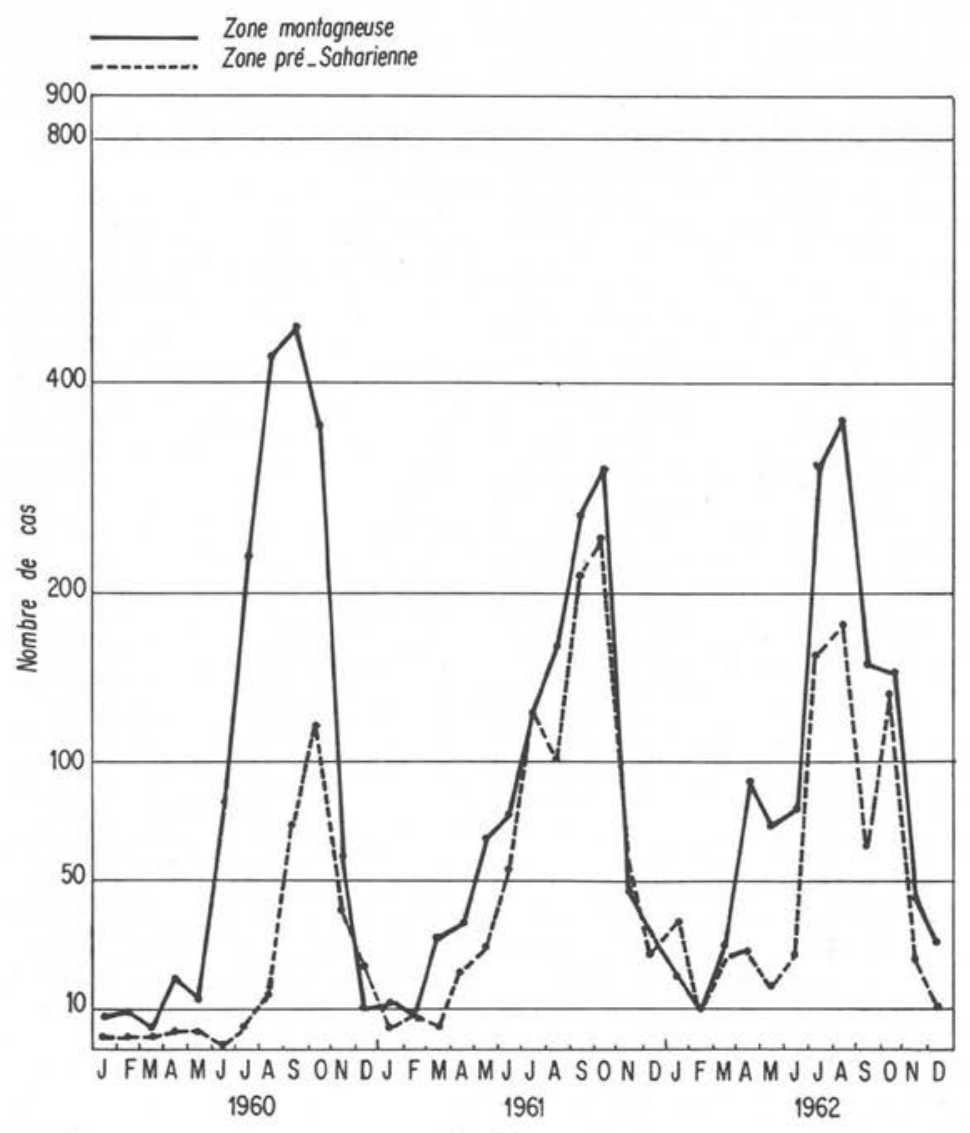

FIG. 22

Traditionnellement, cette zone était considérée comme non ou très peu impaludée. Il n'en est rien et l'on peut se rendre compte à présent que la pénétration y est plus aisée qu'elle est loin d'être indemne (indice systématique à 1,3 pour mille dans la province de Ksar el Souq, à 3,4 pour 1000 , le plus élevé du Maroc, dans la province de Ouarzazate).

Il y a eu 289 cas en 1960 (pour 1220 lames prélevées seulement, le dépistage passif ne fonctionnant réellement d'une façon satisfaisante que depuis 1961 dans cette zone), 900 cas en 1961 (6904 lames prélevées) et 711 cas en 1962 (9 372 lames prélevées) pour une population rurale de 1212022 habitants.

On avait l'habitude de penser que le paludisme apparaissait plus tardivement au cours de l'année que dans le Nord du pays (fig. 20) et en fait c'est bien ce qui s'est passé en 1960: début sensible de l'ascension des cas en juillet et sommet en octobre. Si ce sommet d'octobre se retrouve en 1961 et en 1962, on voit cependant que l'ascen- 
1 Zone cotiere méditerranéenne

2 Zone cotiere otlontique

3 Zone de ploine

4Zone de montogne

5 Zone pré_soharienne

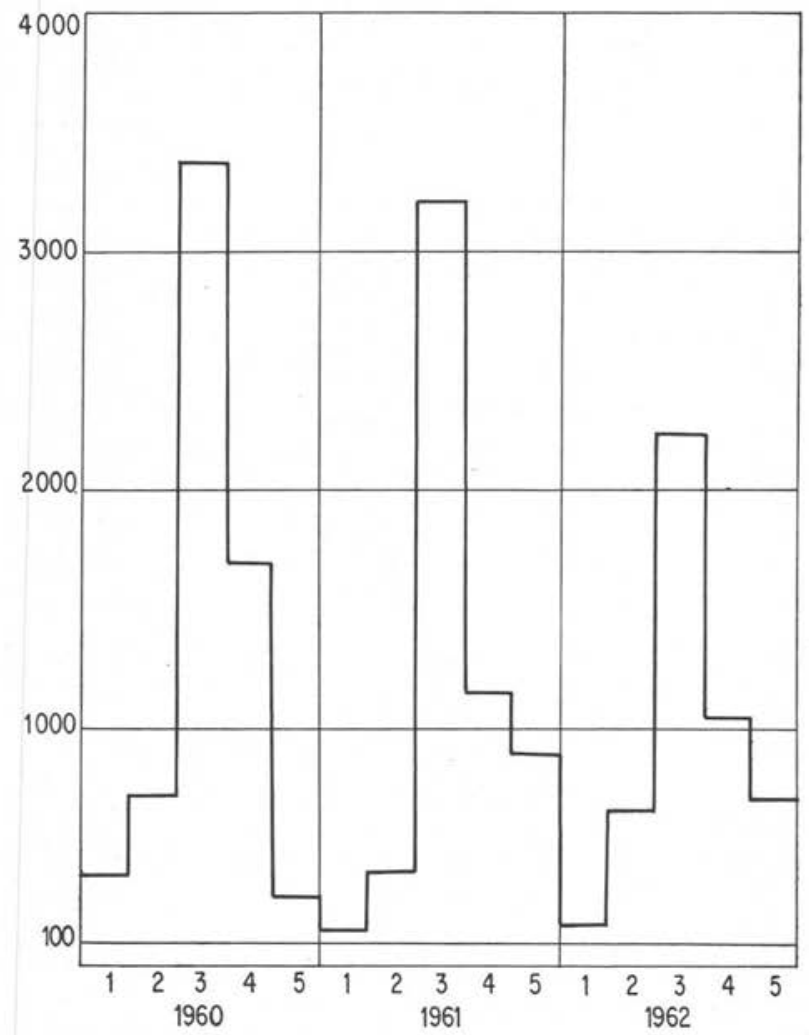

FIG. 23

sion des cas débute dès mai ou juin (sensiblement donc comme dans le reste du pays) et qu'un premier sommet (juillet ou juillet-août) précède le sommet d'octobre. En fait, il est nécessaire de diviser en deux cette zone pré-saharienne : la première comprend les provinces d'Agadir et celle de Ouarzazate, largement ouvertes sur l'Océan dont l'influence se manifeste cependant beaucoup moins que dans le reste du pays, et ce sont elles qui sont responsables du sommet estival d'apparition variable, tandis que le Tafilalet (province de Ksar el Souq), isolé, très continental, très sec et à température estivale très élevée, se montre constant à lui-même d'une année à l'autre et connaît le maximum de cas en octobre (fig. 21). 


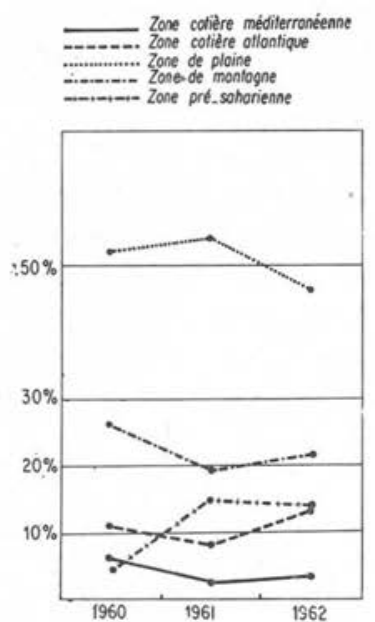

FIG. 24

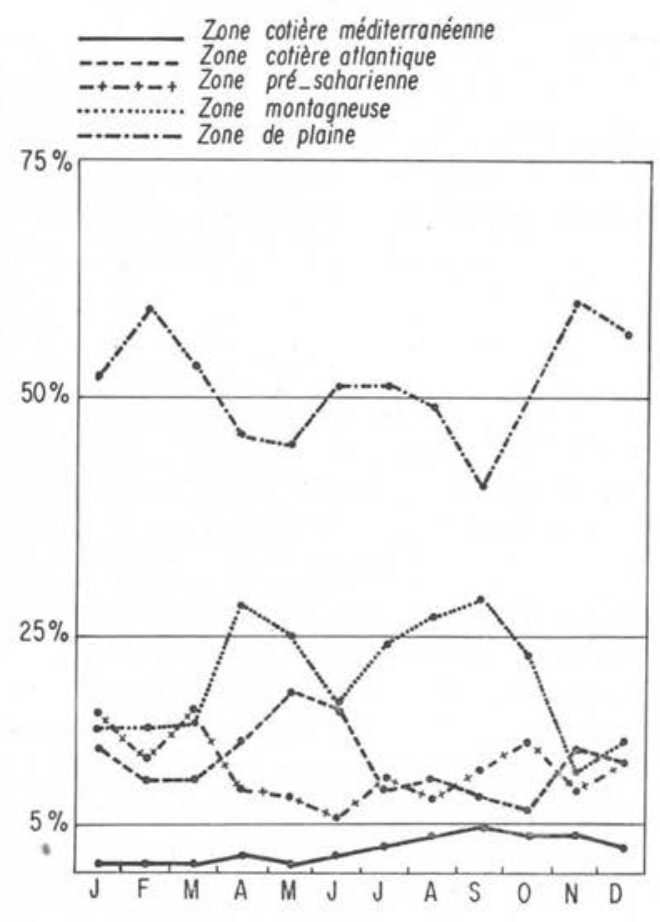

FIg. 26

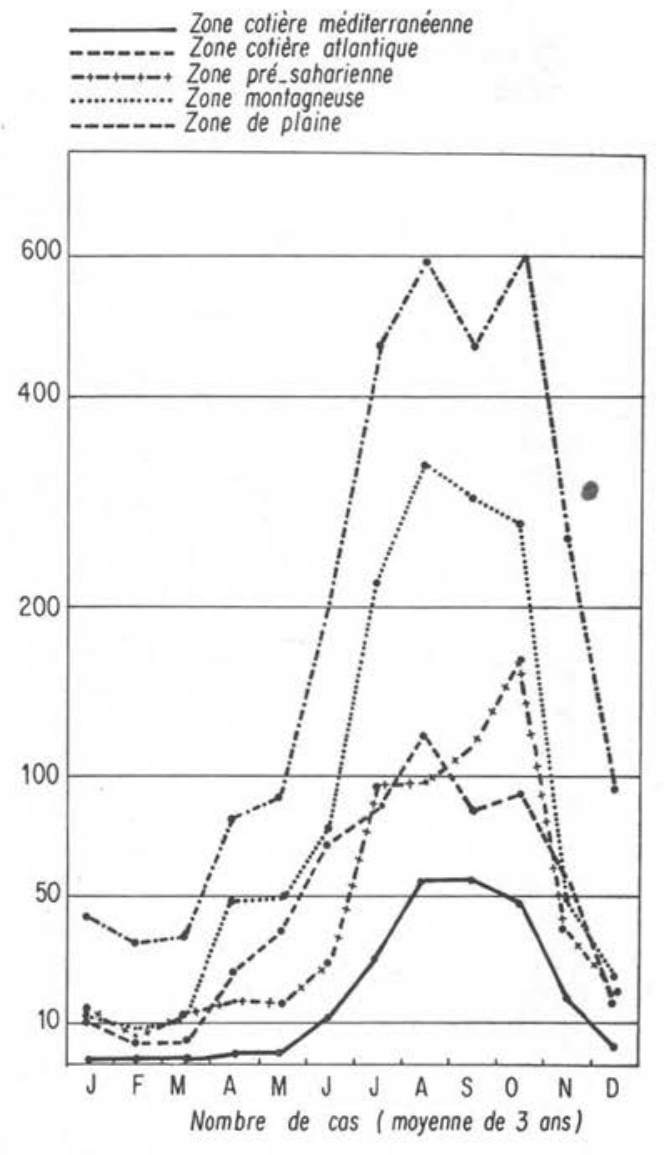

Fig. 25 
Il est cependant certain qu'au cours des trois années précédentes, si la saison du paludisme a été plus courte que dans la plaine par exemple, sa physionomie la rapproche pourtant beaucoup de celle de la montagne (fig. 22).

\section{$* *$}

Certaines conclusions ressortissent de cette étude épidémiologique. Il est évident que le paludisme existe partout au Maroc : aucun cercle (circonscription administrative de 50000 à 200000 personnes en moyenne), qui n'en soit indemne. Il se manifeste d'une façon plus ou moins marquée suivant les régions climatiques (fig. 23 et 24) : la plaine continentale est la plus impaludée, elle est suivie par la zone montagneuse, puis par la zone pré-saharienne, la côte atlantique et enfin la côte méditerranéenne qui est la moins impaludée.

La période de transmission débute en avril ou mai suivant les régions et se termine en octobre.

Il est bien entendu difficile d'établir des moyennes ne portant que sur trois années mais, à titre indicatif, il est cependant possible d'établir un graphique (fig. 25) qui montre le tracé annuel moyen des cas de paludisme suivant les zones et fait ainsi ressortir la physionomie particulière de l'épidémiologie du paludisme suivant les zones climatiques considérées. La figure 26 indique le pourcentage attendu de cas mensuels (moyenne des trois années) suivant ces mêmes zones.

\section{Bibliographie}

1. M. Bonjean, 1947. - L'épidémiologie du paludisme au Maroc. Moncho éd., Rabat.

2. Y. GuY, 1962. - Renseignements fournis par l'étude du rapport larves-adultes d'Anophèles. Ann. Parasitol., 37, 4.

3. G. Houel et F. Donadille, 1963. - Vingt ans de lutte anti-paludique au Maroc. Bull. Inst. Hygiène Maroc, XII, 1-2.

\section{II. - Les Vecteurs}

La faune des Culicidae du pays a été étudiée à différentes reprises (2). Mais il s'est agi lorsque le Maroc a envisagé d'entreprendre l'éradication du paludisme de systématiser les enquêtes entomologiques de telle façon que l'on soit en mesure d'établir une carte faunistique spatiale et temporelle aussi précise que possible et d'acquérir le maximum de renseignements biologiques sur les vecteurs du paludisme.

Dans ce but, 80 gîtes larvaires et 80 stations de capture d'adultes en moyenne ont été désignés dans chacune des 14 provinces du Maroc et chacun de ces points visités à date fixe, une fois par mois, depuis 1959. La technique suivante, très simple, était enseignée aux prospecteurs (Manuel des agents sanitaires ruraux, Direction des Services techniques, Service central d'éradication du paludisme): les pêches se pratiquaient à 
raison de 10 coups de filets d'une minute par gîte et les larves étaient prélevées après chacun de ces 10 coups de filet; dans les stations de capture, après fermeture des portes et des fenêtres, on étendait sur le sol un drap carré de 2,50 $\mathrm{m}$ de côté, un insecticide type Fly-Tox était pulvérisé par 15 coups de pompe et 10 minutes après on procédait à la récolte des adultes.

\section{Anophèles du Maroc}

Les Anophèles du Maroc se répartissent de la façon suivante (3) :

- Sous-genre Anopheles Meigen, 1818.

- Série Anopheles s.s., au sens de G. Senevet (9).

- Anopheles algeriensis Théobald, 1903.

- Anopheles claviger (Meigen, 1804).

- A. claviger petragnanii sahelensis.

- A. claviger missirolii (4).

- Série aitkeni-marteri.

- Anopheles marteri Senevet et Prunelle, 1927.

- Série maculipennis, au sens de G. Senevet, ou sous-genre Maculipennia Buonomini et Mariani, 1945, au sens de J.-A. Rioux (7).

- Anopheles labranchiae Falleroni, 1929.

- Anopheles labranchiae sicaulti Roubaud, 1935.

- Sous-genre Myzorhynchus Blanchard, 1902 (=Cycloleppteron Théobald, 1901).

- Anopheles coustani Laveran, 1900.

- Sous-genre Myzomyia Blanchard, 1902.

- Série Myzomyia s.s., Blanchard, 1902.

- Anopheles sergenti Théobald, 1907.

- Anopheles d'thali Patton, 1905.

- Série Paramyzomyia, au sens de G. Senevet.

- Anopheles hispaniola Théobald, 1903.

- Anopheles multicolor Cambourlin, 1902.

\section{Rôle vecteur et données biologiques sur les différentes espèces}

\section{A. LABRANCHIAE.}

Le vecteur de paludisme le plus important du Maroc est A. labranchiae (ainsi que A. labranchiae sicaulti). Boyd indique (1) qu'en Italie le pourcentage d'infection naturelle des glandes salivaires est de 1,06 . Depuis, l'espèce a été trouvée infectée de sporozoïtes à différentes reprises et dans différents pays. Le nombre de cas de paludisme au Maroc est relativement faible et le nombre de moustiques capturés l'est également, ce qui voue pratiquement à l'échec la recherche de sporozoïtes, même chez $A$. labranchiae, espèce courante et très certainement fortement vectrice. Aussi, est-il souvent nécessaire de recourir à des preuves indirectes et ceci d'autant plus que l'on s'adresse à des espèces moins bien représentées en nombre. 
A. labranchiae est un moustique endophile que l'on trouve fréquemment le matin de bonne heure dans les habitations humaines et animales.

Des tests de précipitine pratiqués ont montré que sur 585 repas, 240 précipitaient au sérum anti de Bovidés (41,0\%), 115 décelaient la présence de sang humain $(19,6 \%)$, 109 celle du sang de cheval $(19,4 \%)$ et le reste, soit $20 \%$, du sang de mouton, de chèvre ou de chien ; de plus, sur ces $20 \%, 16,5 \%$ étaient étiquetés : sang de «mammifères ». Les résultats confirment l'indice de zoophilie trouvé à $66,2 \%$ dans les habitations humaines et à 97,2 \% dans les abris animaux (6). Il apparaît ainsi que l'espèce n'est pas aussi strictement anthropophile qu'on le pensait.

Des tests de sensibilité ont été effectués, soit qu'il s'agisse d'expériences préliminaires au choix d'un insecticide à effet rémanent, soit qu'il s'agisse d'un contrôle après campagne imagicide telle que celle qui est pratiquée depuis 10 ans dans les provinces de Casablanca et Rabat (500 000 personnes protégées). Les lignes de base sont partout normales pour le D.D.T. à $75 \%$. Là où les pulvérisations ont eu lieu depuis quelques années, tout est normal également dans la presque totalité du pays, la concentration léthale 50 (C.L. 50) étant, par exemple, à $1,6 \%$ et la mortalité après exposition d'une heure à la concentration de $4 \%$ de D.D.T. étant de $91 \%$. Cependant, il n'en est pas de même dans une aire localisée du Gharb, au Nord de Rabat, où il semble que soit apparue une certaine résistance de comportement (8). L'évitement aux parois pulvérisées par D.D.T. se confirme d'ailleurs par le fait que l'on ne trouve que peu d'Anophèles dans les locaux pulvérisés et que par contre on en trouve en quantité non négligeable sur les murs enduits de suie de petits cases servant à la cuisine. Dans cette même localisation, on a obtenu en ce qui concerne la Dieldrine une C.L. 50 légèrement inférieure à $0,4 \%$ et seulement une mortalité de $72,0 \%$ après une heure d'exposition à la concentration de $1,6 \%$ de dieldrine ; cette mortalité passe cependant à $99,0 \%$ après 4 heures d'exposition à la même concentration. Une certaine résistance d'A. labranchiae à la dieltrine est donc apparue dans cette région très localisée du Maroc. Elle semble dûe à l'emploi de produits comportant cet insecticide pour la sauvegarde des rizières, nombreuses dans cette plaine. Pour autant que des comparaisons soient possibles avec d'autres espèces résistantes à cet insecticide, il semble bien que dans ce pays le phénomène soit encore mal affirmé et les populations sont composées encore en grande partie d'individus sensibles et d'hybrides; la fraction résistante de la population semble encore assez faible.

La biologie d'A. labranchiae est trop connue pour qu'il soit nécessaire d'y insister. En ce qui concerne les larves, les gîtes sont les classiques « mares à Anophèles » ensoleillées et à végétation (Ranuncula, Potamogeton) et un gîte un peu particulier ici pour l'espèce constitué par les rizières où la densité larvaire est importante. Les adultes sont domestiques et des femelles hibernantes ont été trouvées dans les habitations humaines et animales.

\section{A. SERGENTI.}

Pour ce qui concerne le rôle vecteur du paludisme au Maroc, on peut dire schématiquement que $A$. sergenti joue au Sud du pays le même rôle qu' $A$. labranchiae au 
Nord. Mais le rôle exact est plus difficile à prouver car l'espèce accompagne $A$. labranchiae dans le Sud de l'aire de ce dernier, et $A$. d'thali dans une aire restreinte au Sud du Haut-Atlas. Boyd indique comme pourcentage d'infection naturelle des glandes salivaires : 2,7 en Egypte et 0,5 en Palestine.

Le comportement de ce moustique diffère sensiblement suivant qu'il se rencontre au Nord ou au Sud du Haut-Atlas. Au Ncrd, ainsi que le confirme l'étude du rapport larves/adultes (5), l'espèce est plutôt endophile ; elle est commensale dans les limites nord de son aire avec $A$. labranchiae, espèce reconnue vectrice. Au Sud de la chaîne montagneuse, domaine de villages fortifiés nommés ksour, il existe une différence importante. Les gîtes larvaires, représentés soit par les bords des cours d'eau (Oueds Draa, Dadès et Ziz) plus ou moins salés, soit par des mares souvent très salées le long des oueds, sont riches en larves. Cependant, on ne trouvait jusqu'alors que très peu d'adultes dans les habitations humaines et animales étroitement intriquées dans ces villages fortifiés. On pouvait donc penser qu'il s'agissait d'une espèce exophile, d'autant que ce phénomène coïncidait avec une certaine « exophilie humaine », les habitants couchant pendant tout l'été sur les terrasses plus fraîches que l'intérieur du ksar où il n'est pas exceptionnel de rencontrer des températures nocturnes atteignant ou dépassant $35^{\circ} \mathrm{C}$. Des essais faits sur appât humain et des recherches minutieuses ont montré cependant que si ces moustiques vont effectivement piquer dehors, ils se réfugient ensuite dans un endroit bien particulier : les interstices des toits des rues constitués par un réseau de tiges de bambous ou de troncs de palmier ; là l'humidité est un peu plus marquée et la température est un peu plus basse (de 5 à $9^{\circ} \mathrm{C}$ ). Il semble donc bien que ce gîte de repos à micro-climat particulier serve de refuge à l'espèce qui ne trouve ailleurs que des conditions moins favorables. On peut dire qu' $A$. sergenti a des habitudes alimentaires exophiles et des habitudes de repos endophiles. Il faut noter cependant qu'il demeure relativement endophile lorsqu'un grand nombre d'animaux stabulent à l'intérieur même du village ainsi que cela se rencontre assez fréquemment. C'est pourquoi une campagne de pulvérisations d'insecticide à effet rémanent doit avoir plus de chances de réussite que l'on ne pouvait le craindre avant le début des enquêtes entomologiques précises. Mais comme l'on trouve quand même des spécimens dans les locaux d'habitation (étables en particulier), la couverture totale ne sera assurée que par l'adjonction de pulvérisations sur les murs et les plafonds des rues couvertes à celles pratiquées dans les pièces.

L'anthropophilie de $A$. sergenti est conséquente (20,8 \% dans une expérience de précipitine faite à Zagora, province de Ouarzazate, en août 1961, dans un ksar). Elle ne fait que confirmer le pouvoir vecteur soupçonné de l'espèce au Sud de l'Atlas, alors que dans différentes localités situées au nord de cette chaîne, sur 485 tests pratiqués, un seul à révélé une précipitation au sang humain.

Quelques tests de sensibilité ont été effectués. Ils montrent la parfaite sensibilité d'A. sergenti au D.D.T. comme à la dieldrine, aussi bien au Nord qu'au Sud du HautAtlas.

Les larves ont des gîtes indifféremment héliophiles et scialophiles et la présence de sels dans l'eau, même en quantité importante, ne gêne pas le développement. Les 
adultes se comportent de la curieuse façon indiquée plus haut. Ils sont d'autant plus zoophiles que l'on remonte vers le Nord du pays où existent des troupeaux (bovins, ovins, caprins) de plus en plus importants.

\section{ANOPHELES D'THALI.}

La présence de cette espèce était soupçonnée au Maroc (3). Elle a été confirmée lors d'une mission effectuée en 1959 (10).

C'est également un vecteur très vraisemblable du paludisme au Sud du Haut-Atlas. Il ne semble pas que l'infection naturelle des glandes salivaires ait été fréquemment recherchée ; en tout cas, Boyd ne mentionne rien à ce sujet. Au Maroc, quelques dissections de glandes n'ont pas permis de retrouver de sporozoïtes.

Son anthropophilie est assez conséquente pour indiquer un rôle vecteur non négligeable: un test de précipitine exécuté à Zagora, en septembre 1961 (même lieu que pour le test indiqué concernant $A$. sergenti, mais un mois plus tard), portant sur 160 femelles a montré qu'il s'agissait 34 fois d'un repas de sang humain $(21,2 \%)$.

Cette espèce coexiste avec $A$. sergenti, mais dans une aire plus restreinte qui sera indiquée plus bas. Ce qui a été dit pour le comportement de $A$. sergenti au Sud du HautAtlas concerne également $A$. $d^{\prime}$ thali : même manque d'exigences sur le choix des gîtes larvaires, mêmes gîtes de repos pour les adultes dans les ksour.

\section{ANOPHELES HISPANIOLA.}

A. hispaniola est un mauvais vecteur du paludisme. Cependant, les frères Sergent ont trouvé une fois un porteur de sporozoïtes. Au Maroc, une seule fois également, il est vrai (Midelt, 1948), des sporozoïtes ont été trouvés lors d'une épidémie circonscrite où, de plus, cette espèce représentait la seule existante alors.

Des tests d'anthropophilie ont montré que ce moustique ne se nourrissait sur l'homme que dans $0,6 \%$ des cas. Ces constatations sont heureuses car $A$. hispaniola représente l'espèce la plus répandue du Maroc. Sa répartition géographique est également vaste en Algérie (11).

L'imago est donc zoophile. Il est également exophile en bonne proportion.

Ses gîtes larvaires sont héliophiles de préférence, mais au Sud du Haut-Atlas, il se contente des gîtes peu nombreux (bords des rivières, mares salées) qui satisfont $A$. sergenti et $A$. d'thali.

Des tests de sensibilité pratiqués ont montré une parfaite sensibilité au D.D.T.

\section{ANOPHELES CLAVIGER.}

A. claviger n'existe qu'en petite quantité au Maroc et dans une région très limitée ou, de plus, il coïncide avec $A$. labranchiae. De ce fait, il n'a pu être, ainsi que les suivants, que très peu étudié. Boyd signale qu'il a été trouvé porteur de sporozoïtes à Chypre, en Grèce, en Italie et en Mésopotamie. Peut-être est-il responsab'e des quelques cas de l'automne que l'on rencontre dans la partie orientale du Moyen-Atlas. 
Ses larves aiment les gîtes salés ( 9 à $52 \mathrm{~g}$ de chlorures par litre), mais il faut bien

Ses gîtes larvaires préférentiels se rencontrent en altitude moyenne, mais on en a trouvé à $2500 \mathrm{~m}$. Il s'agit alors le plus souvent de gîtes héliophiles d'eau courante, mais les puits et les citernes peuvent lui convenir en plaine. L'adulte est exophile.

\section{ANOPHELES MULTICOLOR.}

C'est une espèce très régulièrement trouvée dans le Maroc pré-saharien, mais à un très petit nombre d'exemplaires. On la sait vectrice dans les oasis de l'Algérie où cependant elle ne s'est prouvée que peu anthropophile quoique mieux représentée en nombre qu'au Maroc (11).

souligner qu' $A$. sergenti et $A$. d'thali se rencontrent également dans ce même type de gîte qui représente bien souvent le seul possible au Sud du Haut-Atlas.

L'adulte peut piquer l'homme mais semb!e exophile.

\section{ANOPHELES COUSTANI.}

Cette espèce est rare au Maroc. Ses larves préfèrent les marécages et les bords herbeux des grandes mares. L'adulte, contrairement à ce qui a été constaté dans d'autres pays, semble exophile au Maroc.

\section{ANOPHELES MARTERI.}

Cette espèce, rare également au Maroc, se développe en montagne, le long des berges des cours d'eau. L'adulte est exophile.

\section{ANOPHELES ALGERIENSIS.}

C'est l'espèce la plus rare du Maroc de même qu'elle est rare en Algérie (11). Les quelques fois où des larves ont été trouvées, il s'agissait de gîtes d'eau claire en montagne (Moyen-Atlas). L'imago semble exophile.

Ainsi, le pouvoir vecteur de paludisme au Maroc se partage entre A. labranchiae au Nord du pays et $A$. sergenti ainsi que $A$. d'thali au Sud. $A$. claviger joue peut-être un rôle limité et $A$. hispaniola, pourtant le plus répandu, ne semble jouer naturellement aucun rôle.

L'étude de la distribution spatiale et temporelle de ces espèces contribue à expliquer ces faits.

\section{Aires de distribution des principales espèces du Maroc}

\section{A. LABRANCHIAE.}

C'est l'espèce prédominante au Nord du Haut-Atlas.

Les trois premiers mois de l'année voient l'extension progressive d'A. labranchiae 
à partir de deux foyers situés près de Souk-el-Arba et Kénitra, le deuxième persistant pendant tout le trimestre et à partir également de trois foyers formant un triangle SettatChemaïa-Safi, ces trois foyers ne disparaissant jamais au cours de l'année non plus qu'un sixième à Marrakech. En outre, il existe un foyer permanent pendant tout le premier trimestre dans la région Meknès-Fès-Azrou. Le Moyen-Atlas forme à cette période de l'année une barrière naturelle vis-à-vis de l'anophélisme qui est plus tardif sur son versant sud ainsi que le montre l'étude du troisième trimestre.

Pendant le deuxième trimestre, $A$. labranchiae s'installe dans son domaine propre qui ne fera que se préciser pendant le trimestre suivant. Il commence par envahir la totalité du pays entre l'Oum er Rbia et la côte et apparaît dans la région de Berkane et Oujda. Mais il quitte très rapidement la côte atlantique (cette zone est pulvérisée par D.D.T. et c'est une région de marécages qui s'assèchent plus ou moins vite quand cessent les pluies), caractère qui s'accentuera pendant le trimestre suivant. Le territoire occupé d'une façon permanente pendant ce deuxième trimestre forme une bande longeant grosso-modo la côte atlantique et limitée à l'Ouest par la côte elle-même de Tanger à Rabat, puis par une ligne plus continentale joignant Rabat à la moitié de la distance entre El Jadida et Safi ; à l'Est par une ligne joignant Tétuan, Souk-el-Arba, OuedZem, El Kelaa des Shrarna et Marrakech ; la limite sud est représentée par une ligne joignant Marrakech à Essaouira. Les foyers de Beni-Mellal, Fès et Berkane sont également permanents pendant ce trimestre.

Le troisième trimestre connaît l'extension maxima de l'aire de $A$. labranchiae. Le territoire occupé par cette espèce d'une façon permanente est alors représenté par un quadrilatère dont le côté Nord-Est va de Tétuan à Debdou, le côté Sud-Est de Debdou à Marrakech, le côté Ouest de Marrakech à Safi, le dernier côté longeant plus ou moins la côte. Le foyer de Berkane s'englobe dans le foyer principal.

Le quatrième trimestre voit la régression générale de l'aire.

On voit donc que :

- A. labranchiae ne disparaît jamais complètement au cours de l'année dans la moitié Nord-Ouest du pays. Il y persiste constamment des foyers (même en décembre et janvier) situés, pour les premiers et en ce qui concerne le littoral au Sud de Larache et au Sud Kénitra ; il est vraisemblab'e qu'au cours de l'année ce sont les rizières et les cultures maraîchères qui entretiennent là l'anophélisme. Il existe également des foyers près d'El Jadida, Safi et Essaouira et là aussi la persistance de l'anophélisme est dûe en partie aux cultures maraîchères. Entre Meknès, Fès et Azrou, en plus des gîtes habituels, les cultures peuvent également être responsables de l'anophélisme persistant.

- Le foyer oriental n'apparaît qu'en avril, n'est englobé au foyer principal qu'en août et septembre et disparaît en novembre. Il est bien délimité et relativement peu important (sauf pendant les mois d'août et septembre) et disparaît complètement pendant quatre mois de l'année. Ce phénomène n'explique pas à lui seul la disparition presque comp'ète du paludisme dans cette région, mais il contribue à rendre compréhensible l'arrêt de la transmission si l'on pense que l'absence puis le petit nombre de spécimens d'une espèce vectrice coïncide avec l'épuisement du réservoir de virus obtenu par une 
chimioprophylaxie et une chimiothérapie soigneuses prodiguées pendant de nombreuses années dans cette importante région industrielle. De plus, le réservoir de virus n'est plus alimenté par des cas importés d'Algérie dont la frontière était fermée.

- Il faut noter l'apparition de plus en plus tardive de l'anophélisme au fur et à mesure que l'on s'éloigne de la côte pour aller vers l'Est.

- A. labranchiae, espèce vectrice, prédomine dans les régions où la densité humaine est la plus forte. C'est dans son aire que l'on trouve les zones les plus impaludées.

\section{A. SERGENTI.}

Au début du mois de mars, l'anophélisme est réduit au cours supérieur du Draa qui est ainsi le centre d'à partir duquel rayonnera l'une des grandes aires de répartition d'A. sergenti : l'aire située au Sud du Haut-Atlas. Au mois d'avril se dessine le début de la deuxième aire de répartition de l'espèce : celle du Nord du Haut-Atlas, le centre de cette aire étant Marrakech. Le foyer de Berkane naît le mois suivant et ce sera jusqu'en décembre un foyer circonscrit ; ce foyer tellement septentrional s'explique par la position oblique du Sud-Ouest au Nord-Est des chaînes montagneuses, le couloir aride et pré-saharien montant très haut vers la côte méditerranéenne dans la partie orientale du pays.

Les mois de juin, juillet et août voient l'épanouissement de l'aire de l'espèce le long du réseau hydrographique du Sud : Tensift, Draa, Dadès et Ziz. Il faut y ajouter également le cours supérieur et moyen du Souss. Le foyer de Berkane demeure toujours bien localisé et permanent pendant ce trimestre. Un fait curieux est la présence de quelques A. sergenti pendant les mois de juin et juillet (deux années de rang) dans la région de Tanger ; ce phénomène reste inexplicable à moins d'admettre un transport artificiel de quelques spécimens.

Les mois de septembre, octobre et novembre voient l'extension maxima de l'aire de l'espèce qui atteint ses limites extrêmes vers l'Océan Atlantique et vers le Sud. L'aire permanente est alors représentée par une zone qui a pour limite nord une ligne tirée de Chichaoua à Beni-Mellal, puis de cette ville vers Goulmima. La limite sud passe par Foum Zguid et le Sud de Zagora. Le Bassin du Ziz qui avait été le dernier atteint (juillet) se dégage en premier (novembre). Le foyer de Berkane demeure inchangé.

En décembre, janvier et février, l'aire de $A$. sergenti se restreint considérablement et finit par ne plus exister que dans la région de Marrakech. Ce foyer disparaîtra en mars et sera relayé alors par celui du Sud qui renaît.

Pour conclure sur cette espèce, on peut dire que :

- Son aire de répartition est nettement située au Sud du pays et si l'existence des foyers de la région de Berkane est a priori assez étonnante elle s'explique cependant par les conditions géographiques et climatiques semblables à celles du Sud du pays qui font qu'A. sergenti a la possibilité de remonter librement vers le Nord par le couloir de la Moulouya. 
- L'anophélisme à $A$. sergenti est plus tardif que celui à $A$. labranchiae puisqu'il n'existe de façon importante qu'à partir des mois de juin et surtout juillet et que son aire est à son maxima de septembre à novembre. Ce phénomène explique pourquoi le Tafilalet connaît une période de transmission plus tardive que le reste du pays.

- Sur les cartes, les foyers situés au Nord et au Sud du Haut-Atlas paraissent continus de juillet à novembre. En fait, le Haut-Atlas est une barrière géographique assez puissante et élevée pour interdire les communications, ses cols même étant en haute altitude. Il s'agit donc vraisemblablement de part et d'autre de cette chaîne montagneuse de deux populations différentes ne s'interpénétrant pas. Ce fait semble se confir-

mer par les différences de résultats concernant les tests à la précipitine et par la présence exclusive d' $A$. d'thali au Sud de la chaîne $(A$. d'thali ayant là les mêmes caractéristiques biologiques qu' $A$. sergenti).

\section{A. D'THALI.}

Cette espèce n'est pas représentée pour ne pas surcharger la carte. Elle est de découverte plus récente qu' $A$. sergenti avec qui elle coexiste dans une aire localisée : Oueds Ziz et Oued Draa. La période où cette espèce (relativement rare) est la plus courante va d'août à octobre.

A. d'thali est donc une espèce apparaissant tardivement, ne durant pas longtemps et à aire limitée.

\section{A. HISPANIOLA.}

Seul le mois de janvier ne connaît qu'un anophélisme modéré en ce qui concerne cette espèce encore que le foyer de Marrakech soit déjà notablement étendu. Dès février, l'aire de $A$. hispaniola est importante et le Haut-Atlas la divise en deux (des exemplaires ont été trouvés à $2500 \mathrm{~m}$ d'altitude). Les foyers du Nord n'apparaissent que plus tardivement.

L'extension de l'anophélisme à $A$. hispaniola se poursuit pendant le deuxième trimestre mais après un envahissement des cours supérieur et moyen de l'Oum-er-Rbia et du Bou-Regreg, ceux-ci se libèrent en juin et ne seront plus occupés qu'en septembre. Cette périodicité double au cours de l'année est à souligner.

Le troisième trimestre représente l'époque où l'aire de l'espèce est maxima. Elle occupe la quasi-totalité du pays à l'exception de la bande littorale.

Le quatrième trimestre ne voit diminuer que tardivement l'aire de l'espèce. Cependant, les grandes plaines de l'Ouest se libèrent et n'appartiendront plus au domaine de l'espèce qu'au mois de mai.

A. hispaniola est l'espèce marocaine la plus répandue aussi bien en ce qui concerne son aire que son calendrier. On la retrouve en plaine comme en montagne. On en a même retrouvé en été 1962 dans de nombreuses stations de la Hammada du Draa et à l'embouchure du Draa (province de Tarfaya) ce qui représente vraisemblablement les stations les plus au Sud de l'espèce. 

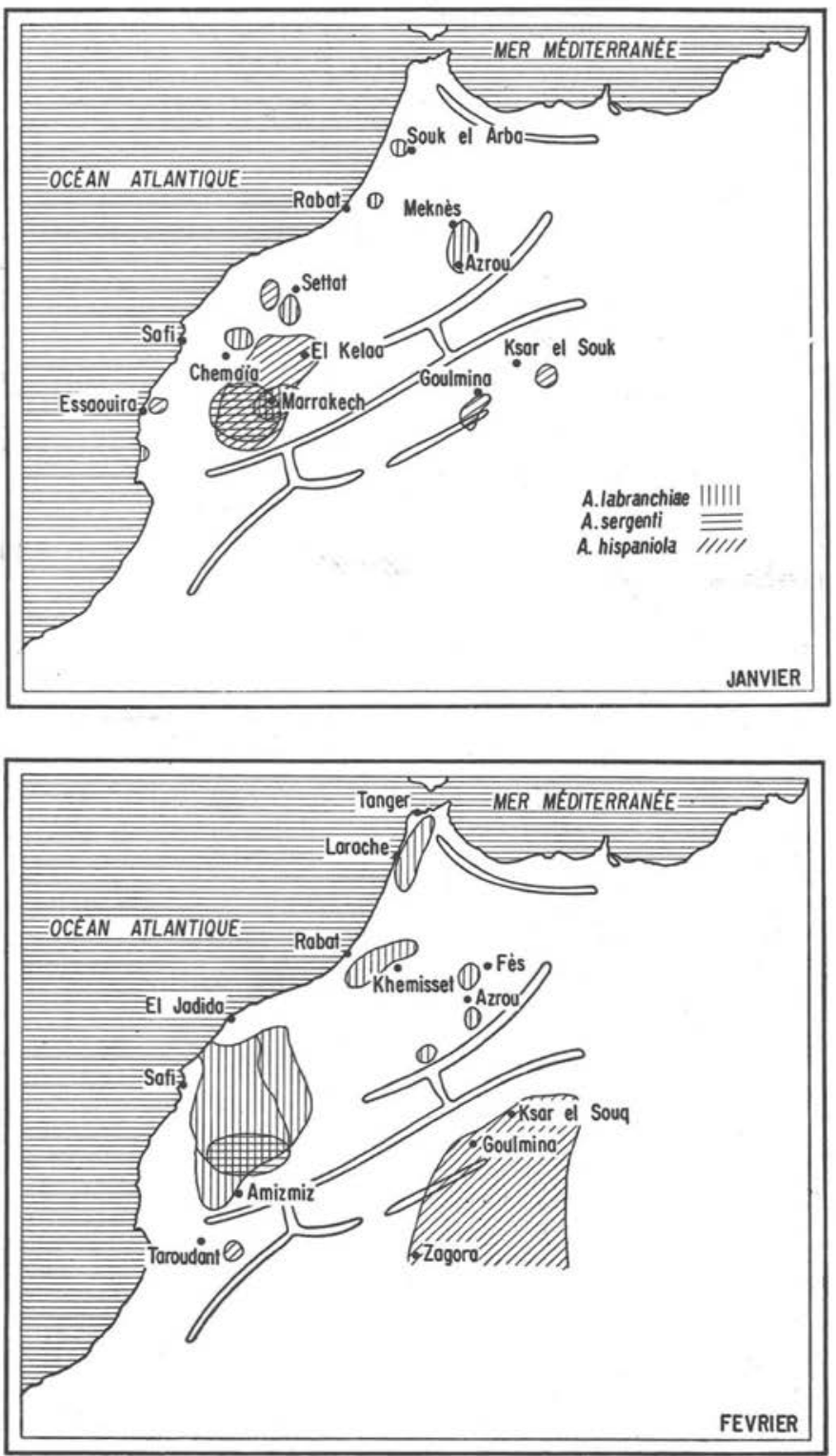

\section{ANOPHELES CLAVIGER.}

C'est un Anophèle de la province de Meknès que l'on trouve particulièrement en montagne (El Hajeb, Ifrane). 

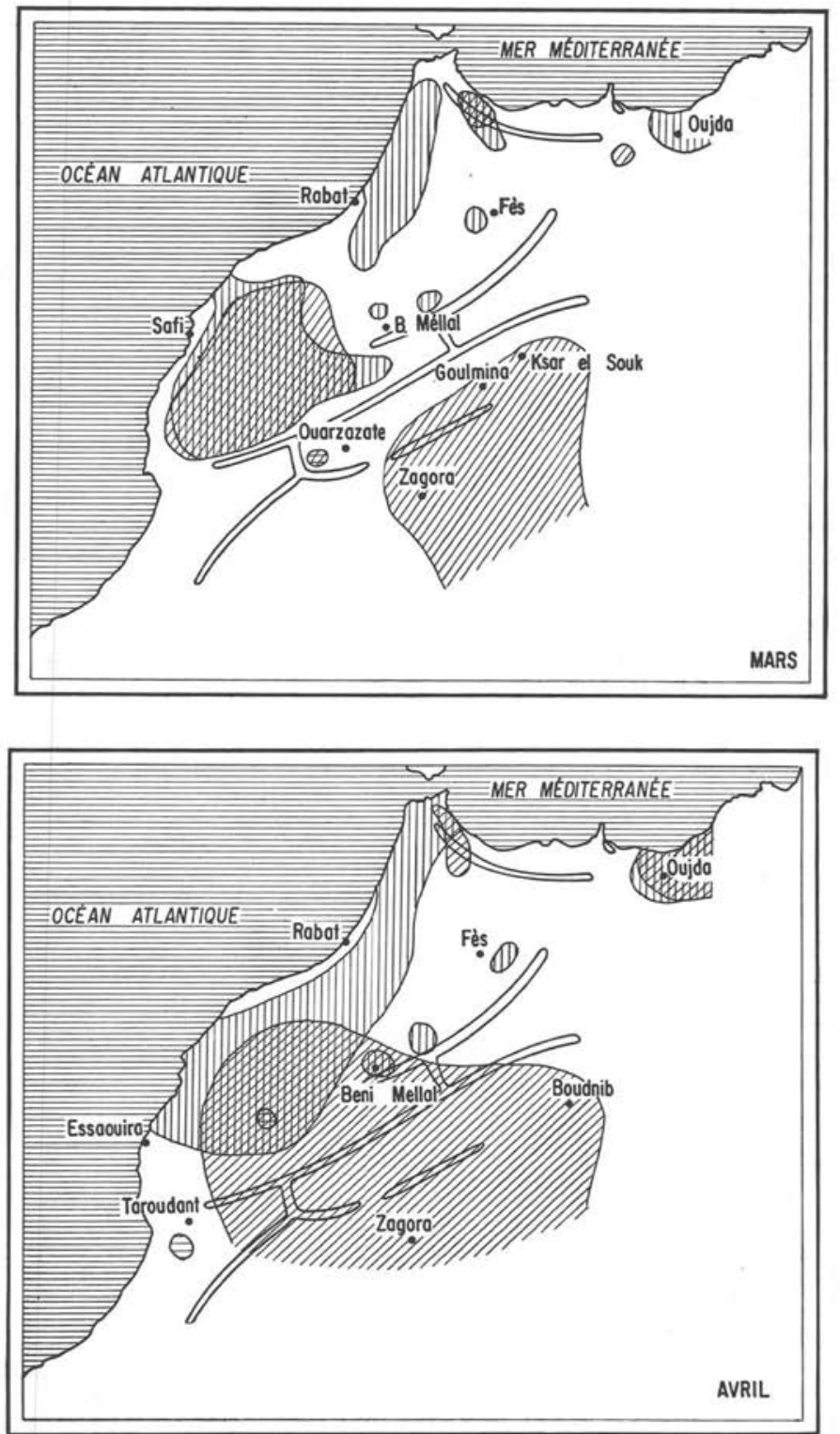

On pêche des larves, en petit nombre mais toute l'année ce qui n'est pas étonnant puisque l'on peut classiquement en trouver même sous la neige ou sous la glace.

Les adultes existent de mai à décembre.

D'autres stations sont connues dans la province de Fès (Taounate en particulier). 

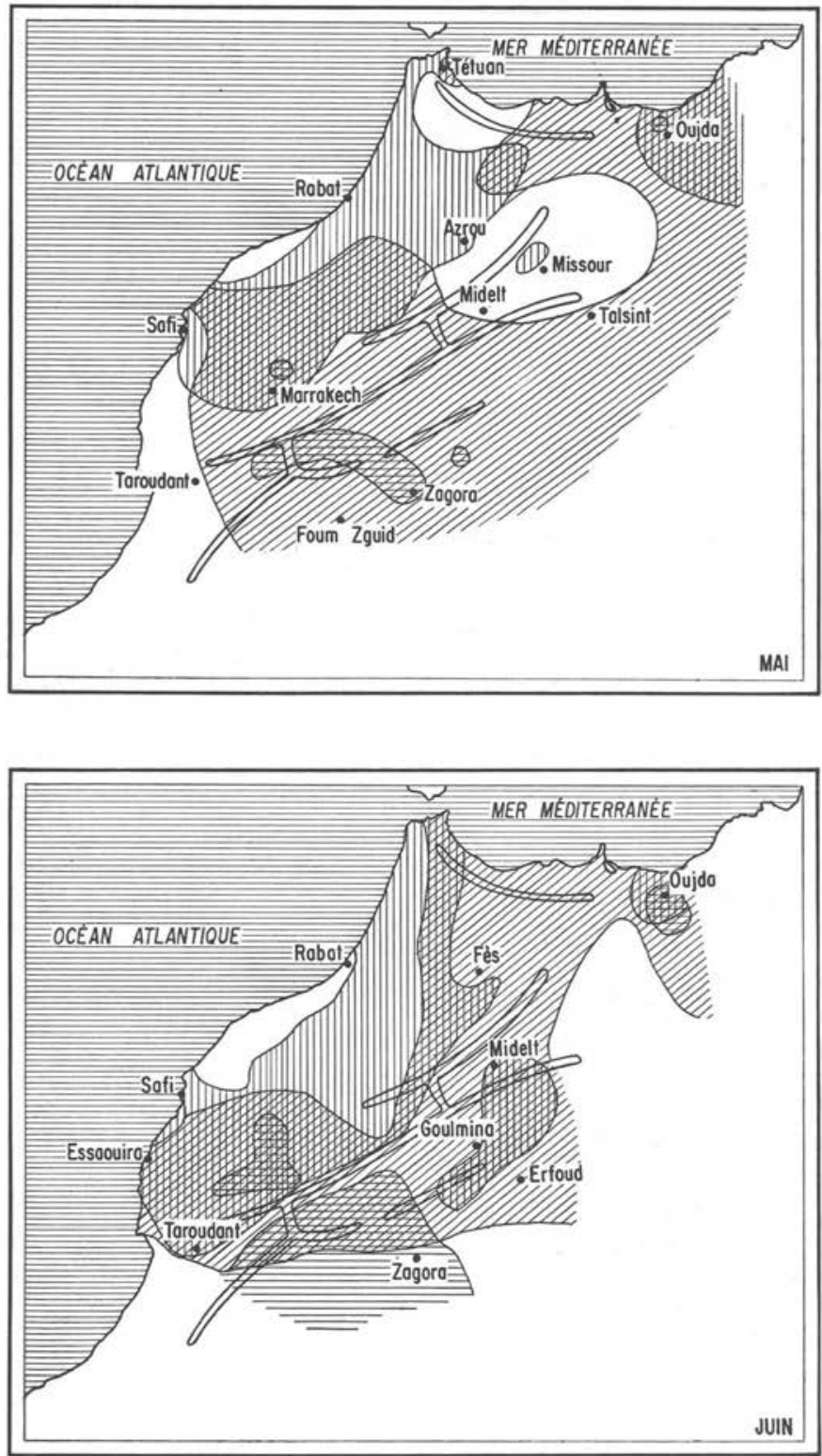

\section{Associations anophéliennes.}

La région de Marrakech est un foyer permanent au cours de l'année pour les trois espèces : $A$. labranchiae, $A$. sergenti et $A$. hispaniola ( $A$. sergenti n'y est absent que pendant le mois de mars). 

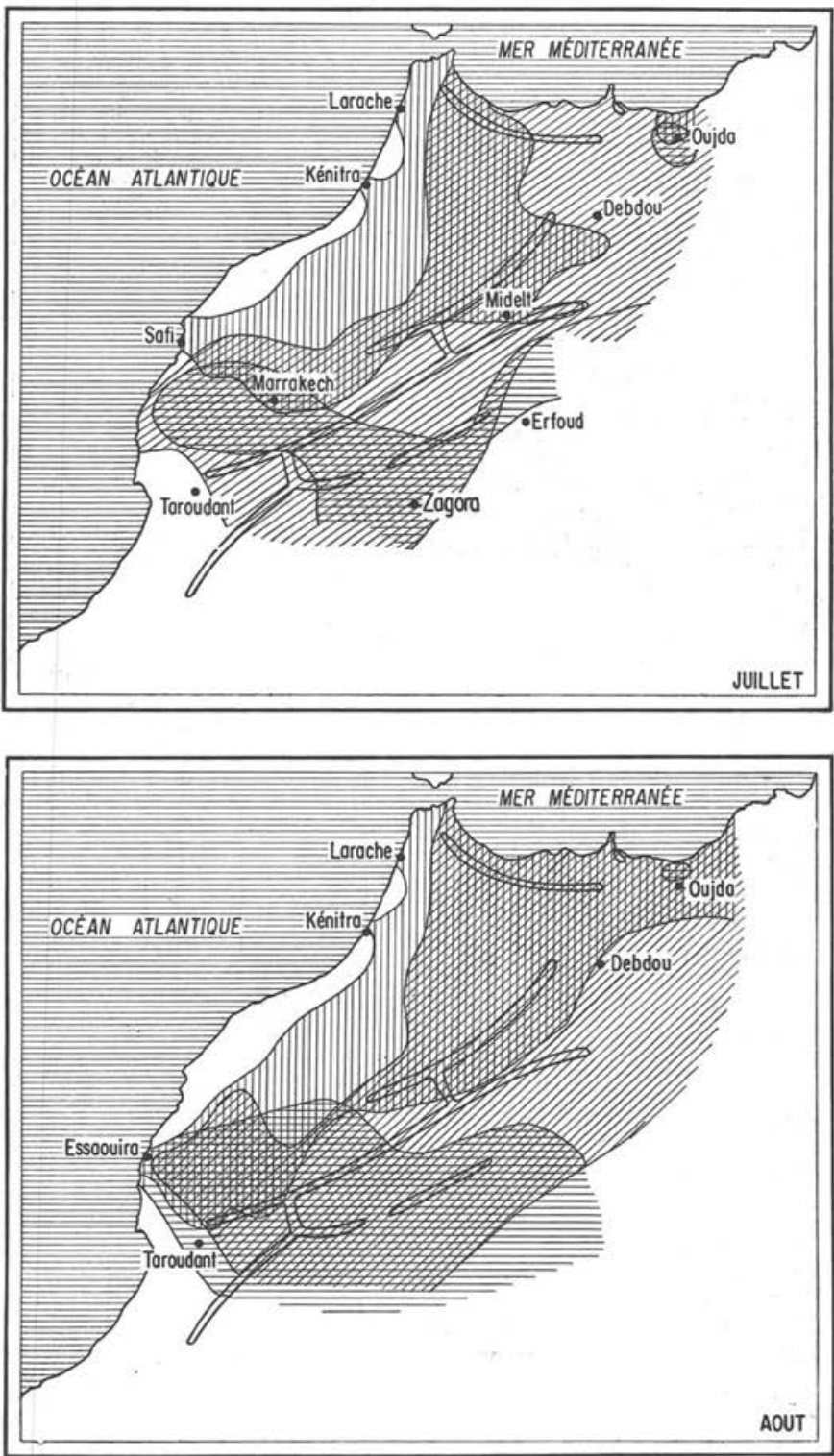

Deux associations sont typiques :

- A. labranchiae $+A$. hispaniola au Nord du Haut-Atlas,

- A. sergenti et $A$. d'thali $+A$. hispaniola au Sud de cette chaîne. 

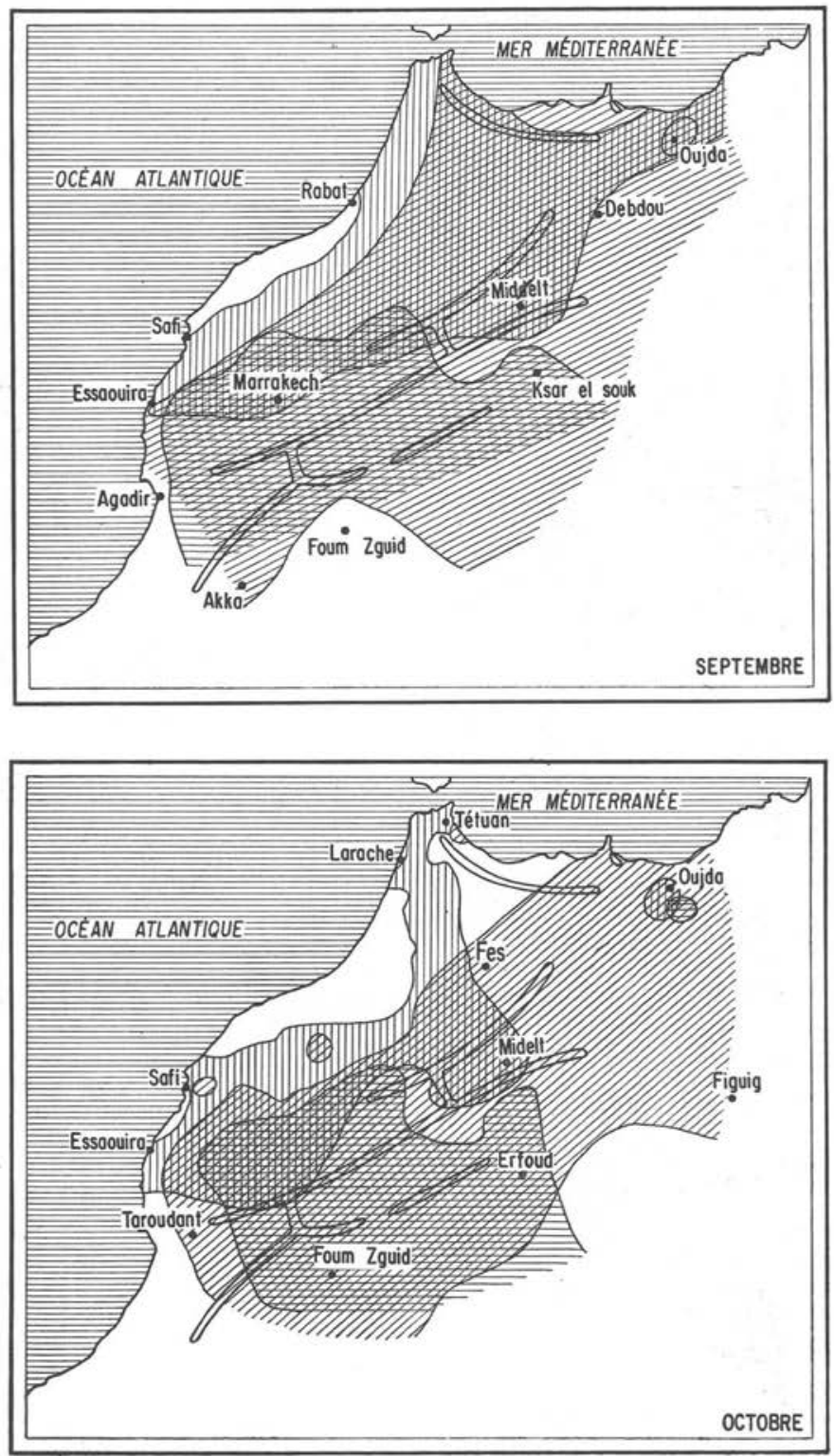

Schématiquement, on peut considérer deux territoires distincts en ce qui concerne l'entomologie du paludisme : celui du Nord du Haut-Atlas, domaine de A. labranchiae et celui du Sud du Haut-Atlas, domaine de $A$. sergenti. 

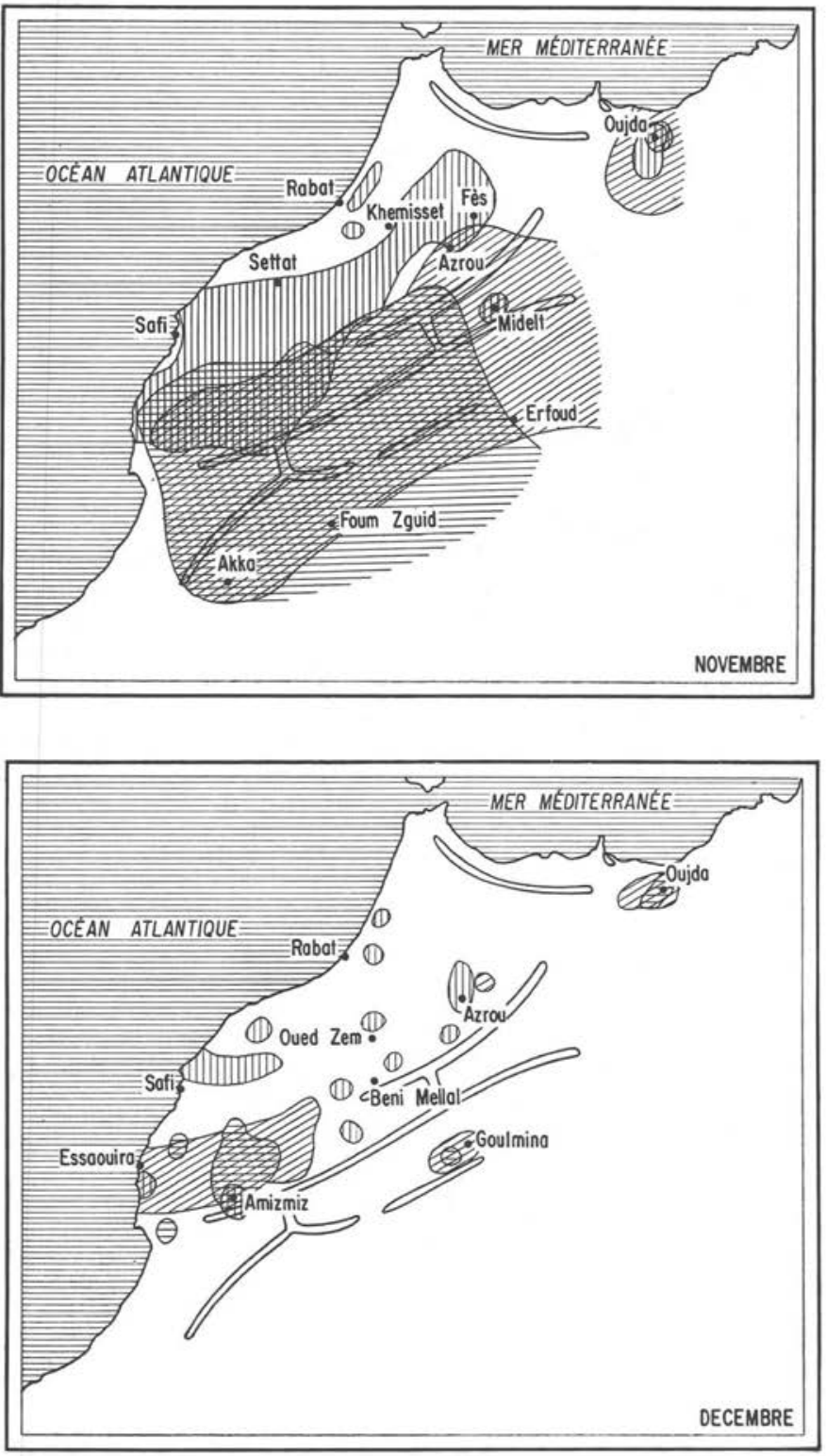


\section{Bibliographie}

1. M. F. BoYD, 1949. - Malariology, Saunders comp., éd., Philadelphie.

2. J. Gaud, 1953. - Notes biogéographiques sur les Culicidés du Maroc. Arch. Inst. Pasteur Maroc, IV, 7.

3. Y. Guy, 1959. - Les Anophèles du Maroc. Mémoire $n^{\circ}$ 7, Soc. Scien. Nat. Phys. Maroc, Rabat.

4. —, 1959. - Les sous-espèces marocaines d'Anopheles claviger Meigen, 1804. Bull. Soc. Scien. Nat. Phys. Maroc, 39.

5. —, 1962. - Renseignements fournis par l'étude du rapport larves-adultes d'Anophèles, Ann. Parasitol., 37, 4.

6. G. Houel et F. Donadille, 1953. - Vingt ans de lutte anti-paludique au Maroc. Bull. Inst. Hyg. Maroc, 12, 1-2.

7. J.-A. Rıoux, 1958. - Les Culicidés du « Midi» méditerranéen. Encyclop. Entomol., P. Lechevalier, éd., Paris.

8. G. SAcCA et Y. GuY, 1960. - Résistance de comportement au D.D.T. chez A. labranchiae au Maroc. Bull. O.M.S., 22.

9. G. Sènevet, 1958. - Les Anophèles du globe. Révision générale. Encyclop. Entomol., P. Lechevalier, éd., Paris.

10. - J. Bernet, J. Clastrier et E. Abonmenc, 1960. - Présence de Anopheles (Myzomyia) d'thali, Patton en deux régions de l'Afrique voisine de l'Atlantique; sa répartition géographique sur le continent africain. Arch. Inst. Pasteur Algérie, 38, 1.

11. - J. Andarelli, 1960. - Contribution à l'étude de la biologie des Moustiques en Algérie et dans le Sahara algérien. Arch. Inst. Pasteur Algérie, 38, 2.

(Laboratoire Central de Parasitologie et d'Entomologie.

Institut National d'Hygiène. Rabat) 